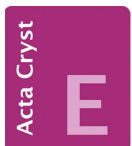

CRYSTALLOGRAPHIC COMMUNICATIONS

ISSN 2056-9890

Received 5 February 2016

Accepted 18 February 2016

Edited by $\mathrm{H}$. Stoeckli-Evans, University of Neuchâtel, Switzerland

Keywords: crystal structure; stilbene; cinnamic acid; photo-acid generator; $\mathrm{O}-\mathrm{H} \cdots \pi$ interactions.

CCDC references: 1454393; 1454392

Supporting information: this article has supporting information at journals.iucr.org/e

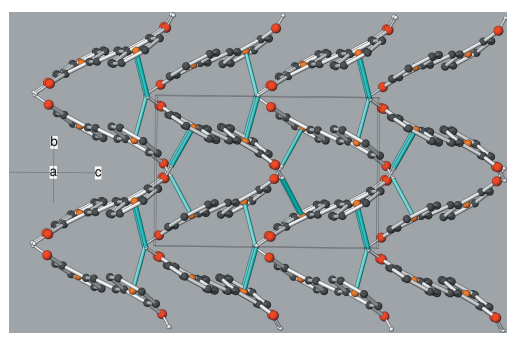

OPEN $\odot$ ACCESS

\section{Investigations of new potential photo-acid generators: crystal structures of 2-[(E)-2-phenyl- ethenyl]phenol (orthorhombic polymorph) and (2E)-3-(2-bromophenyl)-2-phenylprop-2-enoic acid}

\author{
William T. A. Harrison, ${ }^{a *}$ M. John Plater ${ }^{\mathrm{a}}$ and Lee J. Yin ${ }^{\mathrm{b}}$
}

${ }^{\mathbf{a}}$ Department of Chemistry, University of Aberdeen, Meston Walk, Aberdeen AB24 3UE, Scotland, and ${ }^{\mathbf{b}}$ Department of Chemistry, Faculty of Science, University of Malaya, Kuala Lumpur 50603, Malaysia. *Correspondence e-mail: w.harrison@abdn.ac.uk

The title compounds, $\mathrm{C}_{14} \mathrm{H}_{12} \mathrm{O}$, (I), and $\mathrm{C}_{15} \mathrm{H}_{11} \mathrm{BrO}_{2}$, (II), were prepared and characterized as part of our studies of potential new photo-acid generators. In (I), which crystallizes in the orthorhombic space group $P c a 2_{1}$, compared to $P 2_{1} / n$ for the previously known monoclinic polymorph [Cornella \& Martin (2013). Org. Lett. 15, 6298-6301], the dihedral angle between the aromatic rings is $4.35(6)^{\circ}$ and the $\mathrm{OH}$ group is disordered over two sites in a 0.795 (3):0.205 (3) ratio. In the crystal of (I), molecules are linked by $\mathrm{O}-\mathrm{H} \cdots \pi$ interactions involving both the major and minor-OH disorder components, generating [001] chains as part of the herringbone packing motif. The asymmetric unit of (II) contains two molecules with similar conformations (weighted r.m.s. overlay fit = $0.183 \AA$ ). In the crystal of (II), both molecules form carboxylate inversion dimers linked by pairs of $\mathrm{O}-\mathrm{H} \cdots \mathrm{O}$ hydrogen bonds, generating $R_{2}^{2}(8)$ loops in each case. The dimers are linked by pairs of $\mathrm{C}-\mathrm{H} \cdots \mathrm{O}$ hydrogen bonds to form [010] chains.

\section{Chemical context}

Photo-acid generators can be used as additives for creating patterns in a polymer film by irradiation through a mask followed by thermal development and base treatment (Ayothi et al., 2007; Kudo et al., 2008; Steidl et al., 2009). The UV irradiation degrades a small amount of the photo-acid generator in exposed areas, which releases a catalytic amount of a strong acid (commonly triflic acid). This acid subsequently catalyses the degradation of the tert-butylcarboxylate groups of a polymer film in a thermal development step, releasing carboxylic acid groups and isobutene. Treatment with base then solubilizes and removes the degraded polymer film in exposed areas, thereby creating a positive resist image (Ito et al., 1994).

We are exploring new types of organic structures as potential photo-acid generators, which might offer improvements over existing substances. Scheme 1 shows how substituted trans-stilbenes might act as photo-acid generators via sequential photochemical trans-cis isomerization and ringclosing reactions. It should be noted that the photochemical cyclization of stilbenes to phenanthenes in the presence of a hydrogen acceptor such as iodine or propylene oxide is well known (Mallory \& Mallory, 2005). However, in the absence of an oxidant, if a leaving group is present at the ring-closure site, as in structure $\mathbf{3}$, a rapid elimination of HX (structure 5) might 
occur via a stabilized carbocation intermediate 4. In the absence of an oxidant, the cyclized dihydro-phenanthrene compound 6 will equilibrate back to cis-stilbene 2. Stilbenes can also undergo $2 \pi+2 \pi$ photochemical cycloadditions (Fulton \& Dunitz, 1947; Shechter et al., 1963), a possible competing reaction, but the molecular structures and morphology may still favour the desired reaction to proceed in a thin film.
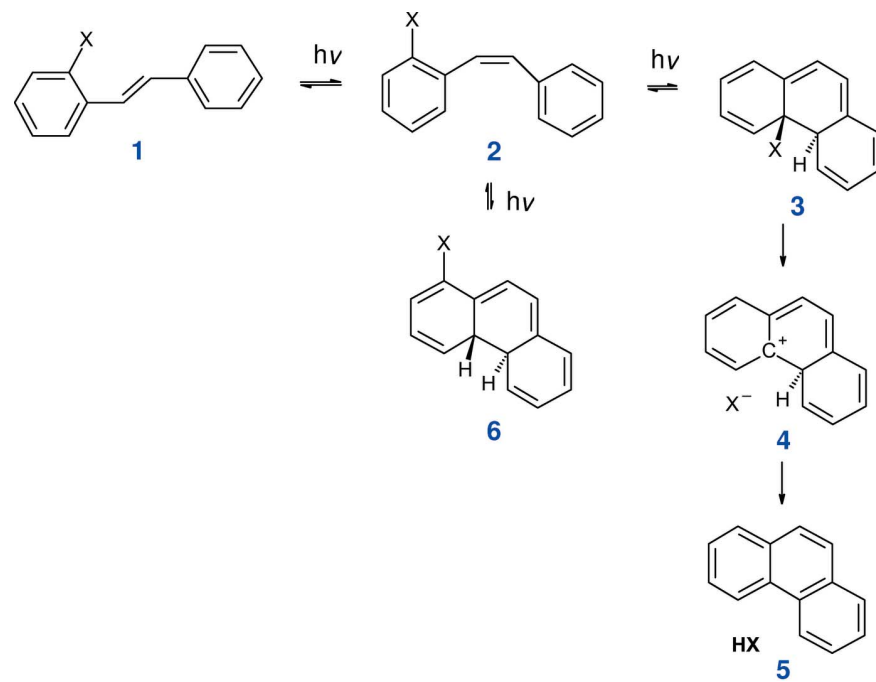

As part of these studies, the syntheses and crystal structures of the title substituted stilbenes, (I) and (II), are now described [compound (II) could also be described as a cinnamic acid derivative: the photochemical reactions of this family of compounds were reported by Schmidt (1971)]. Compound (I) is an intermediate in the synthesis, whereas a close analogue of compound (II) has already been shown to undergo photochemical cyclization to a phenanthrene with concomitant release of $\mathrm{HCl}$ (Geirsson \& Kvaran, 2001). A monoclinic polymorph (space group $P 2_{1} / n$ ) of (I) was reported recently (Cornella \& Martin, 2013) although its crystal structure was not described in detail.<smiles>Oc1ccccc1/C=C/c1ccccc1</smiles>

(I)

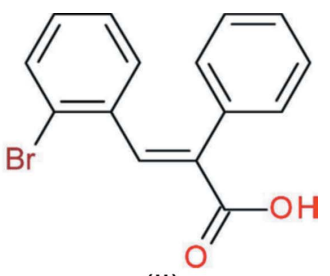

(II)

\section{Structural commentary}

Compound (I) comprises one molecule in the asymmetric unit (Fig. 1), with the $-\mathrm{OH}$ group disordered over two sites in a 0.795 (3):0.205 (3) ratio. For the major disorder component, the $\mathrm{C}_{\mathrm{ar}}-\mathrm{C}_{\mathrm{ar}}-\mathrm{O}-\mathrm{H}(\mathrm{ar}=$ aromatic $)$ torsion angle is $172^{\circ}$. The molecule is close to planar and the dihedral angle between the aromatic rings is $4.35(6)^{\circ}$. The bond lengths of the central unit $[\mathrm{C} 6-\mathrm{C} 7=1.4703$ (19); $\mathrm{C} 7-\mathrm{C} 8=1.3407$ (16); $\mathrm{C} 8-\mathrm{C} 9=$ $1.4720(18) \AA]$ are consistent with data from previous studies

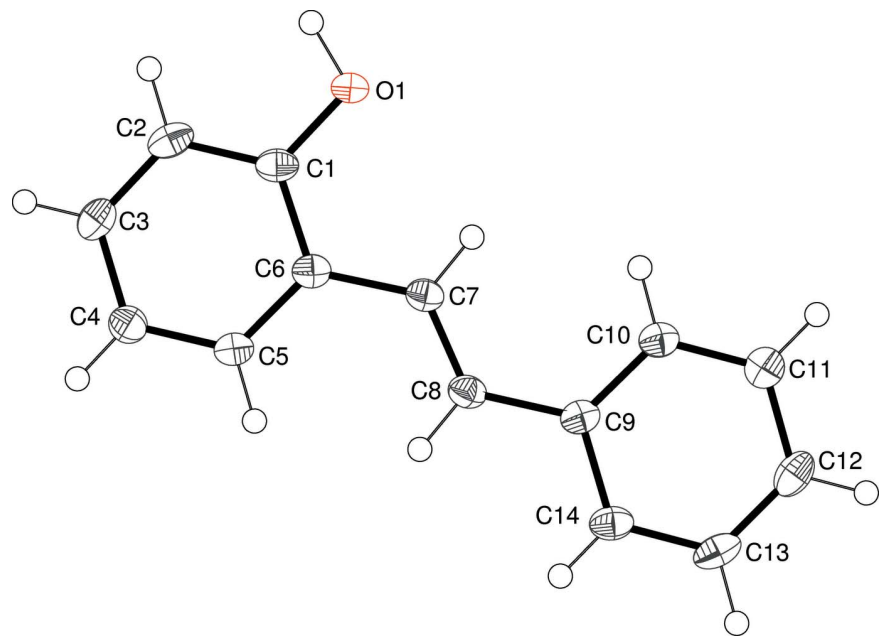

Figure 1

The asymmetric unit of (I), showing $50 \%$ displacement ellipsoids. Only the major disordered component for the $\mathrm{OH}$ group is shown (the minor component is attached to $\mathrm{C} 14$ ).

of similar compounds (Tirado-Rives et al., 1984; Jungk et al., 1984). In the monoclinic polymorph of (I) (Cornella \& Martin, 2013), the asymmetric unit consists of a half-molecule, which is completed by crystallographic inversion symmetry and therefore, of course, the aromatic rings are exactly coplanar: the $\mathrm{OH}$ group is statistically disordered by symmetry and the corresponding $\mathrm{C}-\mathrm{C}-\mathrm{O}-\mathrm{H}$ torsion angle for the monoclinic phase is $-175^{\circ}$.

There are two molecules in the asymmetric unit of (II) (Fig. 2). In the first (C1) molecule, the dihedral angles between the carboxylic acid group and the phenyl and bromobenzene rings are $61.52(6)$ and $55.43(5)^{\circ}$, respectively; the dihedral angle between the aromatic rings is $54.45(5)^{\circ}$. The equivalent data for the second (C16) molecule are 50.72 (6), 60.28 (5) and $61.48(6)^{\circ}$, respectively. The $\mathrm{C} 1$ and $\mathrm{C} 16$ molecules have a similar overall conformation with an r.m.s. deviation of $0.183 \AA$ for the overlay fit for all non-hydrogen atoms. Otherwise, their bond lengths and bond angles are unexceptional and fall within the expected range of values.

\section{Supramolecular features}

The crystal of (I) features $\mathrm{O}-\mathrm{H} \cdots \pi$ interactions as the main supramolecular interaction (Table 1). The major disorder component $(\mathrm{O} 1-\mathrm{H} 1 O)$ generates [001] zigzag chains, as seen

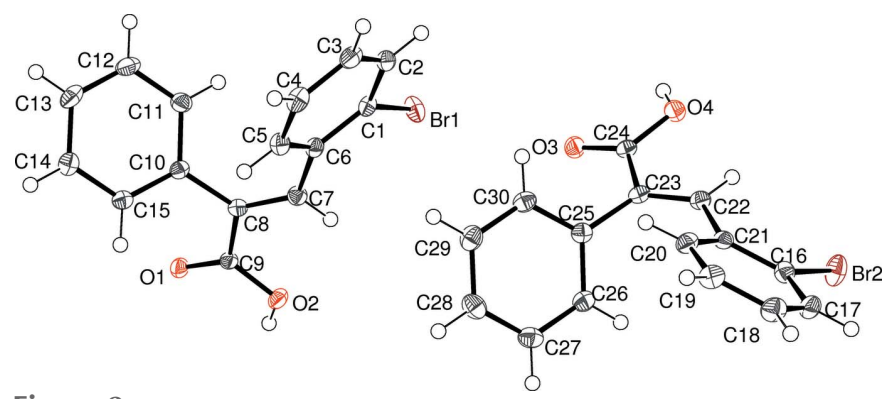

Figure 2

The asymmetric unit of (II), showing 50\% displacement ellipsoids. 
Table 1

Hydrogen-bond geometry $\left(\AA,^{\circ}\right)$ for (I).

$C g 1$ and $C g 2$ are the centroids of rings $\mathrm{C} 1-\mathrm{C} 6$ and $\mathrm{C} 9-\mathrm{C} 14$, respectively.

\begin{tabular}{lllll}
\hline$D-\mathrm{H} \cdots A$ & $D-\mathrm{H}$ & $\mathrm{H} \cdots A$ & $D \cdots A$ & $D-\mathrm{H} \cdots A$ \\
\hline $\mathrm{O} 1-\mathrm{H} 1 \mathrm{\cdots} \cdots C g 2^{\mathrm{i}}$ & 0.98 & 2.66 & $3.5028(13)$ & 144 \\
$\mathrm{O} 2-\mathrm{H} 2 \mathrm{\cdots} \cdots C g 1^{1}$ & 0.91 & 2.74 & $3.646(2)$ & 179 \\
$\mathrm{C} 5-\mathrm{H} 5 \cdots C g 2^{i \mathrm{i}}$ & 0.95 & 2.86 & $3.5337(12)$ & 129 \\
$\mathrm{C} 10-\mathrm{H} 10 \cdots C g 1^{\mathrm{iii}}$ & 0.95 & 2.87 & $3.5742(14)$ & 132 \\
$\mathrm{C} 13-\mathrm{H} 13 \cdots C g 1^{\text {iv }}$ & 0.95 & 2.87 & $3.6015(14)$ & 135 \\
\hline
\end{tabular}

Symmetry codes: (i) $-x+1,-y, z-\frac{1}{2}$; (ii) $x-\frac{1}{2},-y+1$, $z$; (iii) $x+\frac{1}{2},-y, z$; (iv) $-x+1,-y+1, z+\frac{1}{2}$.

Table 2

Hydrogen-bond geometry $\left(\AA,^{\circ}\right)$ for (II).

\begin{tabular}{lllll}
\hline$D-\mathrm{H} \cdots A$ & $D-\mathrm{H}$ & $\mathrm{H} \cdots A$ & $D \cdots A$ & $D-\mathrm{H} \cdots A$ \\
\hline $\mathrm{O} 2-\mathrm{H} 2 O \cdots \mathrm{O} 1^{\mathrm{i}}$ & $0.84(2)$ & $1.80(2)$ & $2.6402(16)$ & $174(2)$ \\
$\mathrm{O} 4-\mathrm{H} 4 O \cdots 3^{\mathrm{ii}}$ & $0.81(2)$ & $1.84(2)$ & $2.6478(16)$ & $178(2)$ \\
$\mathrm{C} 5-\mathrm{H} 5 \cdots 3^{\mathrm{iii}}$ & 0.95 & 2.42 & $3.323(2)$ & 158 \\
$\mathrm{C} 20-\mathrm{H} 20 \cdots \mathrm{O} 1^{\mathrm{iii}}$ & 0.95 & 2.52 & $3.3072(19)$ & 141 \\
\hline
\end{tabular}

Symmetry codes: (i) $\quad-x,-y+1,-z$; $\quad$ (ii) $\quad-x+1,-y+1,-z+1$; $\quad$ (iii) $-x+\frac{1}{2}, y-\frac{1}{2},-z+\frac{1}{2}$.

in Fig. 3. The minor disorder component $(\mathrm{O} 2-\mathrm{H} 2 \mathrm{O})$ also forms [001] chains. There are also some possible very weak $\mathrm{C}-\mathrm{H} \cdots \pi$ interactions. The packing can be described as herringbone when viewed down [100] (Fig. 4). The monoclinic polymorph (Cornella \& Martin, 2013) also features supramolecular chains with the molecules linked by $\mathrm{O}-\mathrm{H} \cdots \pi$

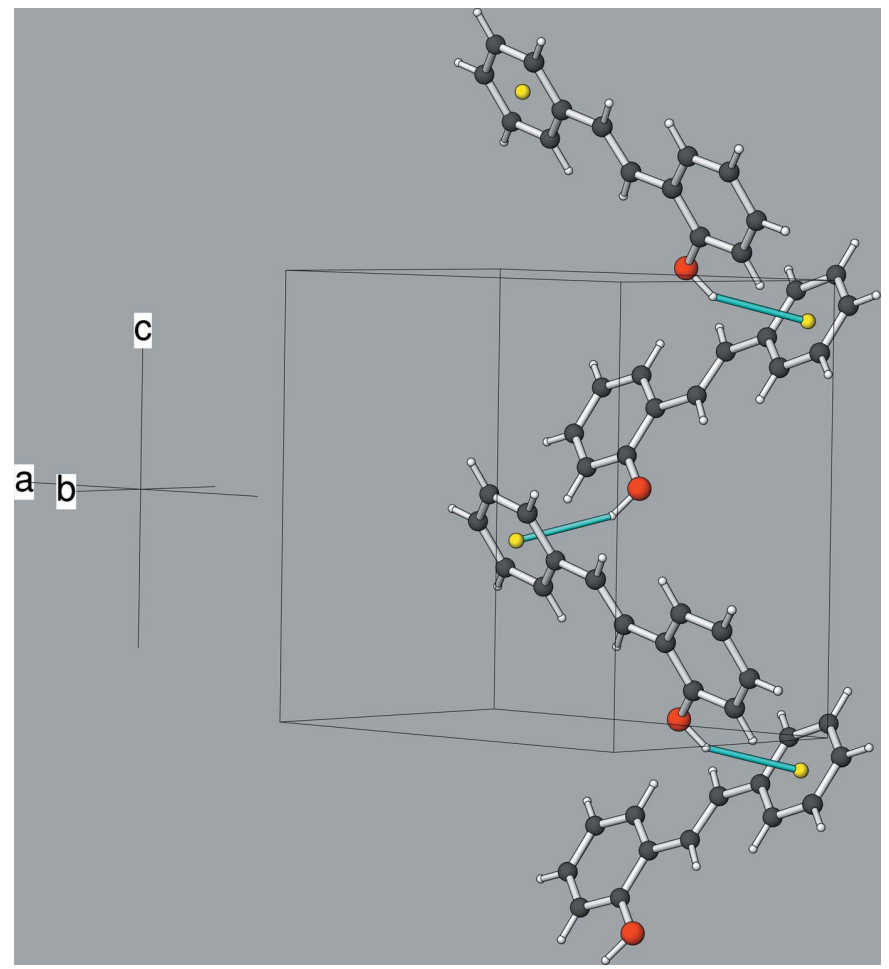

Figure 3

Part of a [001] chain of molecules in the crystal of (I), connected by $\mathrm{O}-$ $\mathrm{H} \cdots \pi$ interactions (cyan lines).

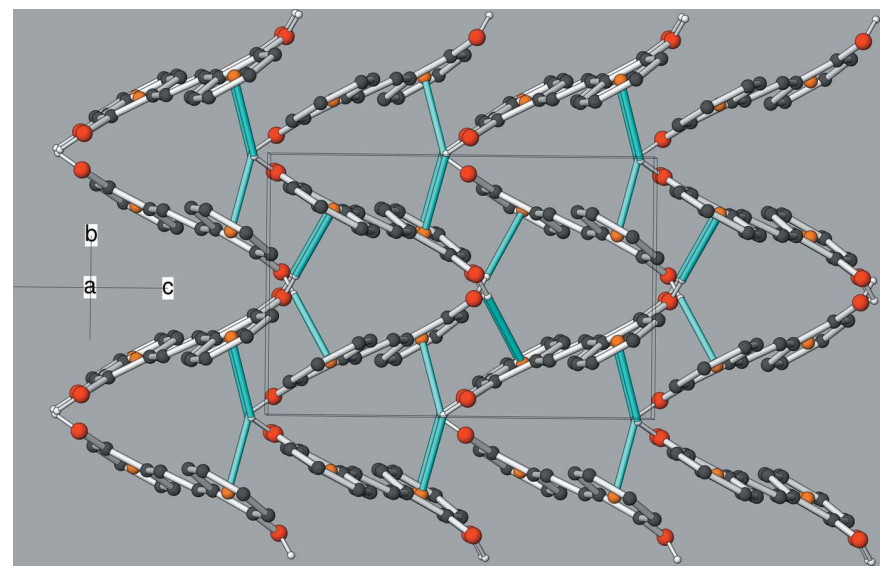

Figure 4

The unit-cell packing in (I), viewed approximately down [100]. The $\mathrm{O}-$ $\mathrm{H} \cdots \pi$ interactions from both disordered components are shown as cyan lines.

interactions but a different overall herringbone packing motif (Fig. 5).

In the crystal of (II), both molecules ( $A$ and $B$ ) form carboxylic acid inversion dimers linked by pairs of $\mathrm{O}-\mathrm{H} \cdots \mathrm{O}$ hydrogen bonds (Table 2), which generate $R_{2}^{2}(8)$ loops in each case. The $(A+A)$ and $(B+B)$ dimers are in turn linked by pairs of $\mathrm{C}-\mathrm{H} \cdots \mathrm{O}$ hydrogen bonds to generate [010] chains (Figs. 6 and 7). This hydrogen-bond scheme is 'balanced,' with both $\mathrm{O} 1$ and $\mathrm{O} 3$ accepting one $\mathrm{O}-\mathrm{H} \cdots \mathrm{O}$ and one $\mathrm{C}-\mathrm{H} \cdots \mathrm{O}$ hydrogen bond. The shortest $\mathrm{Br} \cdot \mathrm{Br}$ contact distance of 3.6504 (4) $\AA$ in the crystal of (II) is slightly shorter than the van der Waals radius sum of $3.70 \AA$ for two $\mathrm{Br}$ atoms (Bondi, 1964).

\section{Database survey}

A survey of the Cambridge Structural Database (Groom \& Allen, 2014) (entries updated to 22 December 2015) revealed ten crystal structures of $E$-2-hydroxy stilbenes with different substituents including (E)-1,2-bis(2-hydroxyphenyl)ethene (refcode CEYKUM; Tirado-Rives et al., 1984), in which the molecules are linked by $\mathrm{O}-\mathrm{H} \cdots \mathrm{O}$ hydrogen bonds. Two substituted $Z$-isomers are also known. A total of 28 analogues

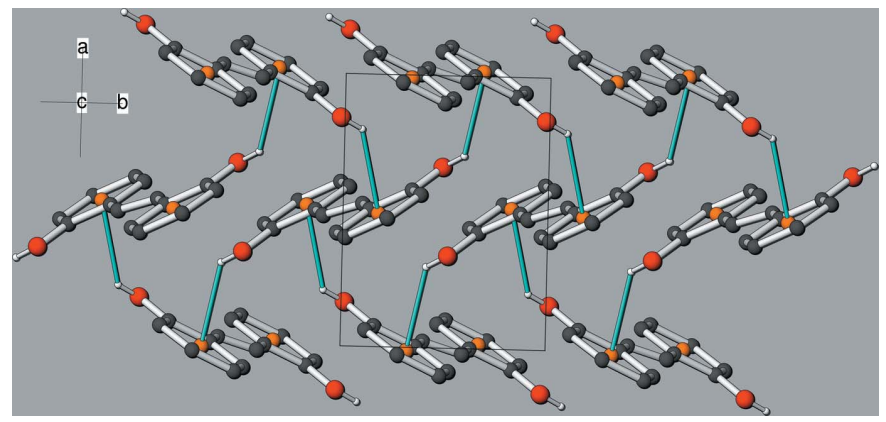

Figure 5

The unit-cell packing in the monoclinic polymorph of $\mathrm{C}_{14} \mathrm{H}_{12} \mathrm{O}$, viewed approximately down [000] (data from Cornella \& Martin, 2013). The O$\mathrm{H} \cdots \pi$ interactions are shown as cyan lines. 


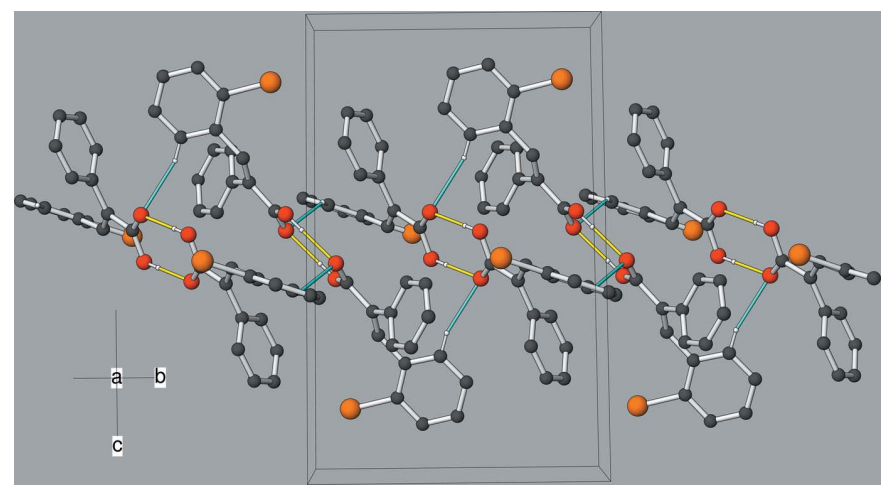

Figure 6

Part of a [010] chain in the crystal of (II), with $\mathrm{O}-\mathrm{H} \cdots$ O hydrogen bonds shown as yellow lines and $\mathrm{C}-\mathrm{H} \cdots \mathrm{O}$ hydrogen bonds shown as cyan lines.

of (II) with different substituents to the aromatic rings were found in the same survey, including the parent compound, 2,3diphenylacrylic acid (refcode OJOFEZ; Fujihara et al., 2011).

\section{Synthesis and crystallization}

Salicylaldehyde $(0.2 \mathrm{~g}, 1.64 \mathrm{mmol})$ and benzyltriphenylphosphonium bromide $(1.0 \mathrm{~g}, 2.31 \mathrm{mmol})$ in dry dimethylformamide (DMF) $(30 \mathrm{ml})$ were treated with sodium methoxide powder $(0.2 \mathrm{~g}, 3.70 \mathrm{mmol})$ and refluxed for $4 \mathrm{~h}$ (Mylona et al., 1986). The reaction mixture was then cooled, acidified with dilute aqueous $\mathrm{HCl}$ and extracted into $\mathrm{CH}_{2} \mathrm{Cl}_{2}$.

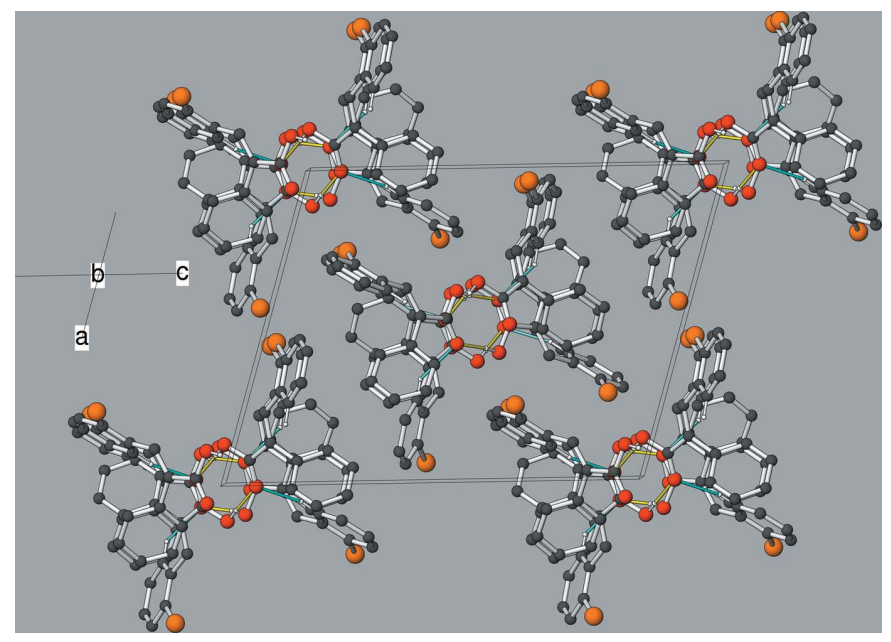

Figure 7

The unit-cell packing in (II), viewed approximately down [010].

The organic layer was washed twice with water to remove DMF, dried over $\mathrm{Na}_{2} \mathrm{SO}_{4}$, concentrated in vacuo and purified by flash chromatography on silica gel. Hexane-diethyl ether (50:50) eluted the title compound (52 mg, 16\%) as a white solid (m.p. 418-419 K), which was recrystallized from hexane/ diethyl ether solution to yield colourless slabs of (I); $m / z \quad 196.0886 \quad\left(M^{+}\right) \quad \mathrm{C}_{14} \mathrm{H}_{12} \mathrm{O}$ requires 196.0883. UV $\lambda_{\max }\left(\mathrm{CHCl}_{3}\right) / \mathrm{nm} 230(\log \varepsilon 4.30), 288$ (4.39) and 315 (4.40). IR $\left(v_{\max } / \mathrm{cm}^{-1}\right) 3528 s, 3019 w, 2923 w, 2852 w, 1585 s, 1498 s, 1454 s$,

Table 3

Experimental details.

(I)

Crystal data

Chemical formula

$M_{\text {r }}$

Crystal system, space group

Temperature (K)

$a, b, c(\AA)$

$\alpha, \beta, \gamma\left({ }^{\circ}\right)$

$V\left(\AA^{3}\right)$

$Z$

Radiation type

$\mu\left(\mathrm{mm}^{-1}\right)$

Crystal size (mm)

Data collection

Diffractometer

Absorption correction

$T_{\min }, T_{\max }$

No. of measured, independent and observed

$[I>2 \sigma(I)]$ reflections

$R_{\text {int }}$

$(\sin \theta / \lambda)_{\max }\left(\AA^{-1}\right)$
$\mathrm{C}_{14} \mathrm{H}_{12} \mathrm{O}$

196.24

Orthorhombic, $P c a 2_{1}$

100

11.6193 (8), 7.6800 (5), 11.3584 (8)

90, 90, 90

1013.58 (12)

4

Mo $K \alpha$

0.08

$0.27 \times 0.16 \times 0.04$

Rigaku CCD

$-$

6984, 2271, 2132

0.031

0.649

$0.034,0.091,1.06$

2271

146

1

$\mathrm{H}$-atom parameters constrained

$0.19,-0.15$
(II)

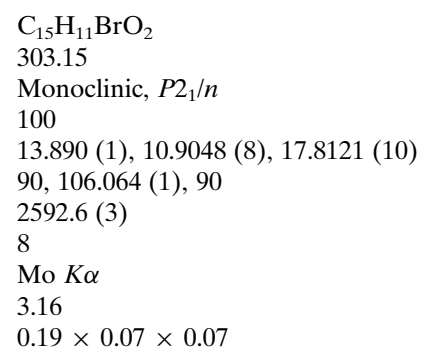

Rigaku CCD

Multi-scan (SADABS; Sheldrick, 2004)

$0.585,0.809$

31964, 5922, 5297

0.035

0.650

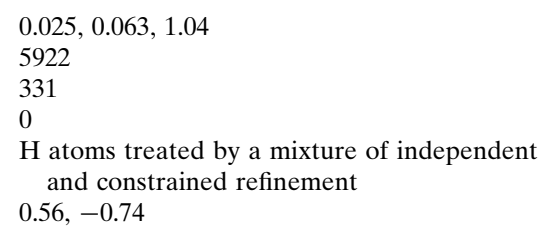

No. of reflections

No. of parameters

$\mathrm{H}$-atom treatment

$\Delta \rho_{\max }, \Delta \rho_{\min }\left(\mathrm{e} \AA^{-3}\right)$

Computer programs: CrystalClear (Rigaku, 2010), SHELXS97 and SHELXL97 (Sheldrick, 2008), ORTEP-3 for Windows (Farrugia, 2012) and ATOMS (Dowty, 1999). 
1332s, 1249s, 1195s, 1088s, 974vs, 845s, 752vs, 724vs, 691vs, 507vs. ${ }^{1} \mathrm{H}$ NMR $\left(400 \mathrm{MHz}, \mathrm{CDCl}_{3}\right) \delta 5.07(1 \mathrm{H}, s), 6.79(1 \mathrm{H}, d, J$ $=8.0), 6.95(1 \mathrm{H}, t, J=7.4), 7.14(2 \mathrm{H}, m), 7.25(1 \mathrm{H}, t, J=6.3)$, $7.35(3 \mathrm{H}, m), 7.52(3 \mathrm{H}, m) .{ }^{13} \mathrm{C} \mathrm{NMR}\left(99.5 \mathrm{MHz}, \mathrm{CDCl}_{3}\right) \delta$ $116.1,121.3,123.1,124.8,126.7,127.3,127.7,128.8,130.3,137.7$ and 153.1 (one resonance is missing).

2-Bromobenzaldehyde $(0.5 \mathrm{~g}, 2.70 \mathrm{mmol})$ and methyl phenylacetate $(0.6 \mathrm{~g}, 4.0 \mathrm{mmol})$ in dry DMF $(30 \mathrm{ml})$ were treated with sodium methoxide powder $(0.3 \mathrm{~g}, 5.6 \mathrm{mmol})$ and refluxed for $4 \mathrm{~h}$. The reaction mixture was then cooled, acidified with dilute aqueous $\mathrm{HCl}$ and extracted into $\mathrm{CH}_{2} \mathrm{Cl}_{2}$. The organic layer was washed twice with water to remove DMF, dried over $\mathrm{Na}_{2} \mathrm{SO}_{4}$, concentrated in vacuo and purified by flash chromatography on silica gel. Hexane-diethyl ether (75:25) eluted (II) (65 mg, 8\%) as a colourless solid, which was recrystallized from hexane/diethyl ether solution as colourless rods. The starting ester was evidently hydrolysed either during the reaction or at the work-up stage; $m / z 300.9866(M+\mathrm{H})$ $\mathrm{C}_{15} \mathrm{H}_{10} \mathrm{O}_{2} \mathrm{Br}$ requires 300.9870 .

\section{Refinement}

Crystal data, data collection and structure refinement details are summarized in Table 3. Atom $\mathrm{H} 1 O$ in (I) was located in a difference Fourier map and refined as riding in its as-found relative position with $U_{\text {iso }}(\mathrm{H})=1.2 U_{\text {eq }}(\mathrm{O})$. The other $\mathrm{H}$ atoms were placed geometrically $(\mathrm{C}-\mathrm{H}=0.95 \AA, \mathrm{O}-\mathrm{H}=0.91 \AA)$ and refined as riding atoms with $U_{\text {iso }}(\mathrm{H})=1.2 U_{\text {eq }}(\mathrm{C}, \mathrm{O})$. The $\mathrm{O}$-bound $\mathrm{H}$ atoms in (II) were located in a difference Fourier map and refined with $U_{\text {iso }}(\mathrm{H})=1.2 U_{\text {eq }}(\mathrm{O})$. The C-bound $\mathrm{H}$ atoms were placed geometrically $(\mathrm{C}-\mathrm{H}=0.95 \AA)$ and refined as riding atoms with $U_{\text {iso }}(\mathrm{H})=1.2 U_{\text {eq }}(\mathrm{C})$.

\section{Acknowledgements}

We thank the EPSRC National Crystallography Service (University of Southampton) for the X-ray data collections and the National Mass Spectrometry Service (University of Swansea) for the high-resolution mass-spectroscopic data.

\section{References}

Ayothi, R., Yi, Y., Cao, H., Yueh, W., Putna, S. \& Ober, C. K. (2007). Chem. Mater. 19, 1434-1444.

Bondi, A. (1964). J. Phys. Chem. 68, 441-451.

Cornella, J. \& Martin, R. (2013). Org. Lett. 15, 6298-6301.

Dowty, E. (1999). ATOMS. Shape Software, Kingsport, Tennessee, USA.

Farrugia, L. J. (2012). J. Appl. Cryst. 45, 849-854.

Fujihara, T., Xu, T., Semba, K., Terao, J. \& Tsuji, Y. (2011). Angew. Chem. Int. Ed. 50, 523-527.

Fulton, J. D. \& Dunitz, J. D. (1947). Nature, 160, 161-162.

Geirsson, J. K. F. \& Kvaran, Á. (2001). J. Photochem. Photobiol. A, 144, 175-177.

Groom, C. R. \& Allen, F. H. (2014). Angew. Chem. Int. Ed. 53, 662671.

Ito, H., Breyta, G., Hofer, D., Sooriyakumaran, R., Petrillo, K. \& Seeger, D. (1994). J. Photopol. Sci. Technol. 7, 433-447.

Jungk, S. J., Fronczek, F. R. \& Gandour, R. D. (1984). Acta Cryst. C40, 1873-1875.

Kudo, H., Watanabe, D., Nishikubo, T., Maruyama, K., Shimizu, D., Kai, T., Shimokawa, T. \& Ober, C. K. (2008). J. Mater. Chem. 18, 3588-3592.

Mallory, F. B. \& Mallory, C. W. (2005). Org. React. 30, 1-456.

Mylona, A., Nikokavouras, J. \& Takakis, I. M. (1986). J. Chem. Res. pp. 433-433.

Rigaku (2010). CrystalClear. Rigaku Inc., Tokyo, Japan.

Schmidt, G. M. J. (1971). Pure Appl. Chem. 27, 647-678.

Shechter, H., Link, W. J. \& Tiers, G. V. D. (1963). J. Am. Chem. Soc. 85, 1601-1605.

Sheldrick, G. M. (2004). SADABS. University of Göttingen, Germany.

Sheldrick, G. M. (2008). Acta Cryst. A64, 112-122.

Steidl, L., Jhaveri, S. J., Ayothi, R., Sha, J., McMullen, J. D., Ng, S. Y., Zipfel, W. R., Zentel, R. \& Ober, C. K. (2009). J. Mater. Chem. 19, 505-513.

Tirado-Rives, J., Oliver, M. A., Fronczek, F. R. \& Gandour, R. D. (1984). J. Org. Chem. 49, 1627-1634. 


\section{supporting information}

Acta Cryst. (2016). E72, 407-411 [doi:10.1107/S2056989016002942]

Investigations of new potential photo-acid generators: crystal structures of 2-[(E)-2-phenylethenyl]phenol (orthorhombic polymorph) and (2E)-3-(2-bromophenyl)-2-phenylprop-2-enoic acid

\section{William T. A. Harrison, M. John Plater and Lee J. Yin}

Computing details

For both compounds, data collection: CrystalClear (Rigaku, 2010); cell refinement: CrystalClear (Rigaku, 2010); data reduction: CrystalClear (Rigaku, 2010); program(s) used to solve structure: SHELXS97 (Sheldrick, 2008); program(s) used to refine structure: SHELXL97 (Sheldrick, 2008); molecular graphics: ORTEP-3 for Windows (Farrugia, 2012) and ATOMS (Dowty, 1999); software used to prepare material for publication: SHELXL97 (Sheldrick, 2008).

(I) 2-[(E)-2-Phenylethenyl]phenol

\section{Crystal data}

$\mathrm{C}_{14} \mathrm{H}_{12} \mathrm{O}$

$M_{r}=196.24$

Orthorhombic, $P c a 2_{1}$

Hall symbol: P 2c -2ac

$a=11.6193(8) \AA$

$b=7.6800(5) \AA$

$c=11.3584(8) \AA$

$V=1013.58(12) \AA^{3}$

$Z=4$

Data collection

Rigaku CCD diffractometer

Radiation source: fine-focus sealed tube Graphite monochromator $\omega$ scans

6984 measured reflections

2271 independent reflections

\section{Refinement}

Refinement on $F^{2}$

Least-squares matrix: full

$R\left[F^{2}>2 \sigma\left(F^{2}\right)\right]=0.034$

$w R\left(F^{2}\right)=0.091$

$S=1.06$

2271 reflections

146 parameters

1 restraint
$F(000)=416$

$D_{\mathrm{x}}=1.286 \mathrm{Mg} \mathrm{m}^{-3}$

Mo $K \alpha$ radiation, $\lambda=0.71073 \AA$

Cell parameters from 7085 reflections

$\theta=2.5-27.5^{\circ}$

$\mu=0.08 \mathrm{~mm}^{-1}$

$T=100 \mathrm{~K}$

Slab, colourless

$0.27 \times 0.16 \times 0.04 \mathrm{~mm}$

2132 reflections with $I>2 \sigma(I)$

$R_{\text {int }}=0.031$

$\theta_{\max }=27.5^{\circ}, \theta_{\min }=2.7^{\circ}$

$h=-15 \rightarrow 13$

$k=-9 \rightarrow 8$

$l=-13 \rightarrow 14$

Primary atom site location: structure-invariant direct methods

Secondary atom site location: difference Fourier map

Hydrogen site location: inferred from neighbouring sites

$\mathrm{H}$-atom parameters constrained 
$w=1 /\left[\sigma^{2}\left(F_{\mathrm{o}}^{2}\right)+(0.0581 P)^{2}+0.0566 P\right]$

where $P=\left(F_{\mathrm{o}}^{2}+2 F_{\mathrm{c}}^{2}\right) / 3$

$(\Delta / \sigma)_{\max }<0.001$

$$
\Delta \rho_{\max }=0.19 \mathrm{e} \AA^{-3}
$$

\section{Special details}

Geometry. All e.s.d.'s (except the e.s.d. in the dihedral angle between two 1.s. planes) are estimated using the full covariance matrix. The cell e.s.d.'s are taken into account individually in the estimation of e.s.d.'s in distances, angles and torsion angles; correlations between e.s.d.'s in cell parameters are only used when they are defined by crystal symmetry. An approximate (isotropic) treatment of cell e.s.d.'s is used for estimating e.s.d.'s involving 1.s. planes.

Refinement. Refinement of $F^{2}$ against ALL reflections. The weighted $R$-factor $w R$ and goodness of fit $S$ are based on $F^{2}$, conventional $R$-factors $R$ are based on $F$, with $F$ set to zero for negative $F^{2}$. The threshold expression of $F^{2}>\sigma\left(F^{2}\right)$ is used only for calculating $R$-factors(gt) etc. and is not relevant to the choice of reflections for refinement. $R$-factors based on $F^{2}$ are statistically about twice as large as those based on $F$, and $R$ - factors based on ALL data will be even larger.

Fractional atomic coordinates and isotropic or equivalent isotropic displacement parameters $\left(\AA^{2}\right)$

\begin{tabular}{|c|c|c|c|c|c|}
\hline & $x$ & $y$ & $z$ & $U_{\text {iso }} * / U_{\text {eq }}$ & Occ. $(<1)$ \\
\hline $\mathrm{C} 1$ & $0.30887(12)$ & $0.13815(16)$ & $0.08810(11)$ & $0.0230(3)$ & \\
\hline H1 & 0.3652 & 0.0929 & 0.0386 & $0.028^{*}$ & $0.205(3)$ \\
\hline $\mathrm{C} 2$ & $0.19331(12)$ & $0.12759(16)$ & $0.05641(11)$ & $0.0258(3)$ & \\
\hline $\mathrm{H} 2$ & 0.1721 & 0.0723 & -0.0152 & $0.031^{*}$ & \\
\hline $\mathrm{C} 3$ & $0.10941(12)$ & $0.19707(16)$ & $0.12852(12)$ & $0.0249(3)$ & \\
\hline H3 & 0.0307 & 0.1890 & 0.1067 & $0.030^{*}$ & \\
\hline $\mathrm{C} 4$ & $0.14037(12)$ & $0.27928(17)$ & $0.23349(12)$ & $0.0244(3)$ & \\
\hline H4 & 0.0829 & 0.3280 & 0.2831 & $0.029^{*}$ & \\
\hline $\mathrm{C} 5$ & $0.25559(12)$ & $0.28929(14)$ & $0.26490(11)$ & $0.0222(3)$ & \\
\hline H5 & 0.2759 & 0.3456 & 0.3364 & $0.027^{*}$ & \\
\hline C6 & $0.34307(12)$ & $0.21877(16)$ & $0.19426(10)$ & $0.0207(3)$ & \\
\hline $\mathrm{C} 7$ & $0.46529(12)$ & $0.22186(16)$ & $0.22775(11)$ & $0.0210(3)$ & \\
\hline H7 & 0.5184 & 0.1701 & 0.1746 & $0.025^{*}$ & \\
\hline $\mathrm{C} 8$ & $0.50832(12)$ & $0.29131(15)$ & $0.32669(11)$ & $0.0219(3)$ & \\
\hline H8 & 0.4550 & 0.3453 & 0.3786 & $0.026^{*}$ & \\
\hline C9 & $0.62995(11)$ & $0.29221(15)$ & $0.36292(11)$ & $0.0203(3)$ & \\
\hline $\mathrm{C} 10$ & $0.71722(12)$ & $0.21042(16)$ & $0.29798(11)$ & $0.0229(3)$ & \\
\hline H10 & 0.6982 & 0.1498 & 0.2277 & $0.027^{*}$ & \\
\hline C11 & $0.83100(13)$ & $0.21675(16)$ & $0.33495(12)$ & $0.0259(3)$ & \\
\hline H11 & 0.8892 & 0.1621 & 0.2893 & $0.031^{*}$ & \\
\hline C12 & $0.86041(12)$ & $0.30333(17)$ & $0.43927(13)$ & $0.0273(3)$ & \\
\hline H12 & 0.9383 & 0.3073 & 0.4646 & $0.033^{*}$ & \\
\hline C13 & $0.77504(12)$ & $0.38320(16)$ & $0.50516(11)$ & $0.0265(3)$ & \\
\hline H13 & 0.7943 & 0.4413 & 0.5763 & $0.032 *$ & \\
\hline C14 & $0.66125(13)$ & $0.37858(16)$ & $0.46739(11)$ & $0.0241(3)$ & \\
\hline H14 & 0.6045 & 0.4340 & 0.5121 & $0.029 *$ & $0.795(3)$ \\
\hline $\mathrm{O} 1$ & $0.39106(10)$ & $0.07105(15)$ & $0.01715(10)$ & $0.0261(3)$ & 0.795 \\
\hline $\mathrm{H} 1 \mathrm{O}$ & 0.3492 & 0.0078 & -0.0443 & $0.031^{*}$ & $0.795(3)$ \\
\hline $\mathrm{O} 2$ & $0.5932(4)$ & $0.4523(7)$ & $0.5351(5)$ & $0.0305(15)$ & $0.205(3)$ \\
\hline $\mathrm{H} 2 \mathrm{O}$ & 0.6381 & 0.5365 & 0.5670 & $0.037^{*}$ & $0.205(3)$ \\
\hline
\end{tabular}


Atomic displacement parameters $\left(\AA^{2}\right)$

\begin{tabular}{lllllll}
\hline & $U^{11}$ & $U^{22}$ & $U^{33}$ & $U^{12}$ & $U^{13}$ & $U^{23}$ \\
\hline C1 & $0.0315(7)$ & $0.0191(5)$ & $0.0183(6)$ & $-0.0012(5)$ & $0.0010(5)$ & $0.0000(4)$ \\
C2 & $0.0358(8)$ & $0.0207(6)$ & $0.0208(6)$ & $-0.0060(5)$ & $-0.0057(5)$ & $0.0003(5)$ \\
C3 & $0.0259(7)$ & $0.0222(6)$ & $0.0266(7)$ & $-0.0043(5)$ & $-0.0061(5)$ & $0.0043(5)$ \\
C4 & $0.0255(7)$ & $0.0231(6)$ & $0.0246(7)$ & $0.0004(5)$ & $0.0012(5)$ & $0.0001(5)$ \\
C5 & $0.0270(6)$ & $0.0207(6)$ & $0.0190(6)$ & $-0.0014(5)$ & $-0.0005(5)$ & $-0.0017(5)$ \\
C6 & $0.0255(6)$ & $0.0171(5)$ & $0.0194(6)$ & $-0.0026(5)$ & $0.0011(5)$ & $0.0005(4)$ \\
C7 & $0.0245(6)$ & $0.0204(6)$ & $0.0180(6)$ & $-0.0004(5)$ & $0.0025(5)$ & $-0.0005(4)$ \\
C8 & $0.0240(7)$ & $0.0207(6)$ & $0.0208(6)$ & $-0.0026(5)$ & $0.0045(5)$ & $-0.0022(4)$ \\
C9 & $0.0260(7)$ & $0.0182(6)$ & $0.0166(6)$ & $-0.0040(5)$ & $-0.0001(5)$ & $0.0025(4)$ \\
C10 & $0.0275(7)$ & $0.0213(6)$ & $0.0199(6)$ & $-0.0030(5)$ & $0.0005(5)$ & $0.0003(4)$ \\
C11 & $0.0276(7)$ & $0.0233(6)$ & $0.0269(7)$ & $-0.0001(5)$ & $-0.0015(5)$ & $0.0031(5)$ \\
C12 & $0.0285(7)$ & $0.0238(7)$ & $0.0296(8)$ & $-0.0056(5)$ & $-0.0102(6)$ & $0.0072(5)$ \\
C13 & $0.0388(8)$ & $0.0225(6)$ & $0.0184(6)$ & $-0.0084(5)$ & $-0.0072(6)$ & $0.0017(5)$ \\
C14 & $0.0330(7)$ & $0.0207(6)$ & $0.0185(6)$ & $-0.0038(5)$ & $0.0014(5)$ & $0.0001(5)$ \\
O1 & $0.0247(6)$ & $0.0328(7)$ & $0.0209(6)$ & $0.0027(5)$ & $-0.0004(4)$ & $-0.0101(5)$ \\
O2 & $0.027(3)$ & $0.035(3)$ & $0.030(3)$ & $-0.004(2)$ & $0.003(2)$ & $-0.014(2)$ \\
& & & & & & \\
\hline
\end{tabular}

Geometric parameters $\left(\AA,{ }^{\circ}\right)$

\begin{tabular}{llll}
\hline $\mathrm{C} 1-\mathrm{O} 1$ & $1.3517(17)$ & $\mathrm{C} 8-\mathrm{H} 8$ & 0.9500 \\
$\mathrm{C} 1-\mathrm{C} 2$ & $1.3925(18)$ & $\mathrm{C} 9-\mathrm{C} 10$ & $1.4024(17)$ \\
$\mathrm{C} 1-\mathrm{C} 6$ & $1.4126(18)$ & $\mathrm{C} 9-\mathrm{C} 14$ & $1.4073(18)$ \\
$\mathrm{C} 1-\mathrm{H} 1$ & 0.9300 & $\mathrm{C} 10-\mathrm{C} 11$ & $1.388(2)$ \\
$\mathrm{C} 2-\mathrm{C} 3$ & $1.381(2)$ & $\mathrm{C} 10-\mathrm{H} 10$ & 0.9500 \\
$\mathrm{C} 2-\mathrm{H} 2$ & 0.9500 & $\mathrm{C} 11-\mathrm{C} 12$ & $1.401(2)$ \\
$\mathrm{C} 3-\mathrm{C} 4$ & $1.3963(19)$ & $\mathrm{C} 11-\mathrm{H} 11$ & 0.9500 \\
$\mathrm{C} 3-\mathrm{H} 3$ & 0.9500 & $\mathrm{C} 12-\mathrm{C} 13$ & $1.386(2)$ \\
$\mathrm{C} 4-\mathrm{C} 5$ & $1.388(2)$ & $\mathrm{C} 12-\mathrm{H} 12$ & 0.9500 \\
$\mathrm{C} 4-\mathrm{H} 4$ & 0.9500 & $\mathrm{C} 13-\mathrm{C} 14$ & $1.390(2)$ \\
$\mathrm{C} 5-\mathrm{C} 6$ & $1.4036(18)$ & $\mathrm{C} 13-\mathrm{H} 13$ & 0.9500 \\
$\mathrm{C} 5-\mathrm{H} 5$ & 0.9500 & $\mathrm{C} 14-\mathrm{O} 2$ & $1.240(5)$ \\
$\mathrm{C} 6-\mathrm{C} 7$ & $1.4703(19)$ & $\mathrm{C} 14-\mathrm{H} 14$ & 0.9340 \\
$\mathrm{C} 7-\mathrm{C} 8$ & $1.3407(16)$ & $\mathrm{O} 1-\mathrm{H} 1 \mathrm{O}$ & 0.9794 \\
$\mathrm{C} 7-\mathrm{H} 7$ & 0.9500 & $\mathrm{O} 2-\mathrm{H} 2 \mathrm{O}$ & \\
$\mathrm{C} 8-\mathrm{C} 9$ & $1.4720(18)$ & & 116.8 \\
& & & 116.8 \\
$\mathrm{O} 1-\mathrm{C} 1-\mathrm{C} 2$ & $120.33(12)$ & $\mathrm{C} 7-\mathrm{C} 8-\mathrm{H} 8$ & $117.89(12)$ \\
$\mathrm{O} 1-\mathrm{C} 1-\mathrm{C} 6$ & $118.51(12)$ & $\mathrm{C} 9-\mathrm{C} 8-\mathrm{H} 8$ & $123.03(11)$ \\
$\mathrm{C} 2-\mathrm{C} 1-\mathrm{C} 6$ & $121.17(12)$ & $\mathrm{C} 10-\mathrm{C} 9-\mathrm{C} 14$ & $119.08(12)$ \\
$\mathrm{O} 1-\mathrm{C} 1-\mathrm{H} 1$ & 0.7 & $\mathrm{C} 10-\mathrm{C} 9-\mathrm{C} 8$ & $120.92(12)$ \\
$\mathrm{C} 2-\mathrm{C} 1-\mathrm{H} 1$ & 120.0 & $\mathrm{C} 14-\mathrm{C} 9-\mathrm{C} 8$ & 119.5 \\
$\mathrm{C} 6-\mathrm{C} 1-\mathrm{H} 1$ & 118.8 & $\mathrm{C} 11-\mathrm{C} 10-\mathrm{C} 9$ & 119.5 \\
$\mathrm{C} 3-\mathrm{C} 2-\mathrm{C} 1$ & $120.33(11)$ & $\mathrm{C} 11-\mathrm{C} 10-\mathrm{H} 10$ & $120.32(13)$ \\
$\mathrm{C} 3-\mathrm{C} 2-\mathrm{H} 2$ & 119.8 & $\mathrm{C} 9-\mathrm{C} 10-\mathrm{H} 10$ & $\mathrm{C} 10-\mathrm{C} 11-\mathrm{C} 12$ \\
$\mathrm{C} 1-\mathrm{C} 2-\mathrm{H} 2$ & 119.8 & & \\
& & &
\end{tabular}




\begin{tabular}{|c|c|c|c|}
\hline $\mathrm{C} 2-\mathrm{C} 3-\mathrm{C} 4$ & $119.97(13)$ & $\mathrm{C} 10-\mathrm{C} 11-\mathrm{H} 11$ & 119.8 \\
\hline $\mathrm{C} 2-\mathrm{C} 3-\mathrm{H} 3$ & 120.0 & $\mathrm{C} 12-\mathrm{C} 11-\mathrm{H} 11$ & 119.8 \\
\hline $\mathrm{C} 4-\mathrm{C} 3-\mathrm{H} 3$ & 120.0 & $\mathrm{C} 13-\mathrm{C} 12-\mathrm{C} 11$ & $119.49(13)$ \\
\hline $\mathrm{C} 5-\mathrm{C} 4-\mathrm{C} 3$ & $119.55(13)$ & $\mathrm{C} 13-\mathrm{C} 12-\mathrm{H} 12$ & 120.3 \\
\hline $\mathrm{C} 5-\mathrm{C} 4-\mathrm{H} 4$ & 120.2 & $\mathrm{C} 11-\mathrm{C} 12-\mathrm{H} 12$ & 120.3 \\
\hline $\mathrm{C} 3-\mathrm{C} 4-\mathrm{H} 4$ & 120.2 & $\mathrm{C} 12-\mathrm{C} 13-\mathrm{C} 14$ & $120.18(12)$ \\
\hline $\mathrm{C} 4-\mathrm{C} 5-\mathrm{C} 6$ & $122.03(12)$ & $\mathrm{C} 12-\mathrm{C} 13-\mathrm{H} 13$ & 119.9 \\
\hline $\mathrm{C} 4-\mathrm{C} 5-\mathrm{H} 5$ & 119.0 & $\mathrm{C} 14-\mathrm{C} 13-\mathrm{H} 13$ & 119.9 \\
\hline $\mathrm{C} 6-\mathrm{C} 5-\mathrm{H} 5$ & 119.0 & $\mathrm{O} 2-\mathrm{C} 14-\mathrm{C} 13$ & $113.8(3)$ \\
\hline $\mathrm{C} 5-\mathrm{C} 6-\mathrm{C} 1$ & $116.96(12)$ & $\mathrm{O} 2-\mathrm{C} 14-\mathrm{C} 9$ & $125.0(3)$ \\
\hline $\mathrm{C} 5-\mathrm{C} 6-\mathrm{C} 7$ & $123.05(11)$ & $\mathrm{C} 13-\mathrm{C} 14-\mathrm{C} 9$ & $121.19(13)$ \\
\hline $\mathrm{C} 1-\mathrm{C} 6-\mathrm{C} 7$ & $119.98(11)$ & $\mathrm{C} 13-\mathrm{C} 14-\mathrm{H} 14$ & 119.4 \\
\hline $\mathrm{C} 8-\mathrm{C} 7-\mathrm{C} 6$ & $125.69(12)$ & $\mathrm{C} 9-\mathrm{C} 14-\mathrm{H} 14$ & 119.4 \\
\hline $\mathrm{C} 8-\mathrm{C} 7-\mathrm{H} 7$ & 117.2 & $\mathrm{C} 1-\mathrm{O} 1-\mathrm{H} 1 \mathrm{O}$ & 105.2 \\
\hline $\mathrm{C} 6-\mathrm{C} 7-\mathrm{H} 7$ & 117.2 & $\mathrm{C} 14-\mathrm{O} 2-\mathrm{H} 2 \mathrm{O}$ & 101.9 \\
\hline $\mathrm{C} 7-\mathrm{C} 8-\mathrm{C} 9$ & $126.46(12)$ & & \\
\hline $\mathrm{O} 1-\mathrm{C} 1-\mathrm{C} 2-\mathrm{C} 3$ & $-179.60(12)$ & $\mathrm{C} 7-\mathrm{C} 8-\mathrm{C} 9-\mathrm{C} 10$ & $-3.09(18)$ \\
\hline $\mathrm{C} 6-\mathrm{C} 1-\mathrm{C} 2-\mathrm{C} 3$ & $0.32(18)$ & $\mathrm{C} 7-\mathrm{C} 8-\mathrm{C} 9-\mathrm{C} 14$ & $176.96(11)$ \\
\hline $\mathrm{C} 1-\mathrm{C} 2-\mathrm{C} 3-\mathrm{C} 4$ & $0.36(19)$ & $\mathrm{C} 14-\mathrm{C} 9-\mathrm{C} 10-\mathrm{C} 11$ & $-0.88(17)$ \\
\hline $\mathrm{C} 2-\mathrm{C} 3-\mathrm{C} 4-\mathrm{C} 5$ & $-0.47(19)$ & $\mathrm{C} 8-\mathrm{C} 9-\mathrm{C} 10-\mathrm{C} 11$ & $179.16(12)$ \\
\hline $\mathrm{C} 3-\mathrm{C} 4-\mathrm{C} 5-\mathrm{C} 6$ & $-0.10(19)$ & $\mathrm{C} 9-\mathrm{C} 10-\mathrm{C} 11-\mathrm{C} 12$ & $0.94(19)$ \\
\hline $\mathrm{C} 4-\mathrm{C} 5-\mathrm{C} 6-\mathrm{C} 1$ & $0.74(17)$ & $\mathrm{C} 10-\mathrm{C} 11-\mathrm{C} 12-\mathrm{C} 13$ & $-0.22(19)$ \\
\hline $\mathrm{C} 4-\mathrm{C} 5-\mathrm{C} 6-\mathrm{C} 7$ & $-177.62(12)$ & $\mathrm{C} 11-\mathrm{C} 12-\mathrm{C} 13-\mathrm{C} 14$ & $-0.54(19)$ \\
\hline $\mathrm{O} 1-\mathrm{C} 1-\mathrm{C} 6-\mathrm{C} 5$ & $179.07(11)$ & $\mathrm{C} 12-\mathrm{C} 13-\mathrm{C} 14-\mathrm{O} 2$ & $178.9(3)$ \\
\hline $\mathrm{C} 2-\mathrm{C} 1-\mathrm{C} 6-\mathrm{C} 5$ & $-0.85(17)$ & $\mathrm{C} 12-\mathrm{C} 13-\mathrm{C} 14-\mathrm{C} 9$ & $0.59(19)$ \\
\hline $\mathrm{O} 1-\mathrm{C} 1-\mathrm{C} 6-\mathrm{C} 7$ & $-2.52(17)$ & $\mathrm{C} 10-\mathrm{C} 9-\mathrm{C} 14-\mathrm{O} 2$ & $-178.0(3)$ \\
\hline $\mathrm{C} 2-\mathrm{C} 1-\mathrm{C} 6-\mathrm{C} 7$ & $177.56(11)$ & $\mathrm{C} 8-\mathrm{C} 9-\mathrm{C} 14-\mathrm{O} 2$ & $2.0(4)$ \\
\hline $\mathrm{C} 5-\mathrm{C} 6-\mathrm{C} 7-\mathrm{C} 8$ & $-0.48(19)$ & $\mathrm{C} 10-\mathrm{C} 9-\mathrm{C} 14-\mathrm{C} 13$ & $0.12(18)$ \\
\hline $\mathrm{C} 1-\mathrm{C} 6-\mathrm{C} 7-\mathrm{C} 8$ & $-178.79(11)$ & $\mathrm{C} 8-\mathrm{C} 9-\mathrm{C} 14-\mathrm{C} 13$ & $-179.92(11)$ \\
\hline $\mathrm{C} 6-\mathrm{C} 7-\mathrm{C} 8-\mathrm{C} 9$ & $178.55(12)$ & & \\
\hline
\end{tabular}

Hydrogen-bond geometry $\left(\AA,{ }^{\circ}\right)$

$\mathrm{Cg} 1$ and $\mathrm{Cg} 2$ are the centroids of rings $\mathrm{C} 1-\mathrm{C} 6$ and $\mathrm{C} 9-\mathrm{C} 14$, respectively.

\begin{tabular}{lllll}
\hline$D-\mathrm{H} \cdots A$ & $D-\mathrm{H}$ & $\mathrm{H} \cdots A$ & $D \cdots A$ & $D-\mathrm{H} \cdots A$ \\
\hline $\mathrm{O} 1-\mathrm{H} 1 \mathrm{o} \cdots C g 2^{\mathrm{i}}$ & 0.98 & 2.66 & $3.5028(13)$ & 144 \\
$\mathrm{O} 2-\mathrm{H} 2 \mathrm{o} \cdots C g 1$ & 0.91 & 2.74 & $3.646(2)$ & 179 \\
$\mathrm{C} 5-\mathrm{H} 5 \cdots C g 2^{\mathrm{ii}}$ & 0.95 & 2.86 & $3.5337(12)$ & 129 \\
$\mathrm{C} 10-\mathrm{H} 10 \cdots C g 1^{\mathrm{iii}}$ & 0.95 & 2.87 & $3.5742(14)$ & 132 \\
$\mathrm{C} 13-\mathrm{H} 13 \cdots C g 1^{\mathrm{iv}}$ & 0.95 & 2.87 & $3.6015(14)$ & 135 \\
\hline
\end{tabular}

Symmetry codes: (i) $-x+1,-y, z-1 / 2$; (ii) $x-1 / 2,-y+1, z$; (iii) $x+1 / 2,-y, z$; (iv) $-x+1,-y+1, z+1 / 2$.

(II) (2E)-3-(2-Bromophenyl)-2-phenylprop-2-enoic acid

Crystal data

$\mathrm{C}_{15} \mathrm{H}_{11} \mathrm{BrO}_{2}$

$M_{r}=303.15$

Monoclinic, $P 2_{1} / n$

Hall symbol: -P $2 \mathrm{yn}$ 


$$
\begin{aligned}
& a=13.890(1) \AA \\
& b=10.9048(8) \AA \\
& c=17.8121(10) \AA \\
& \beta=106.064(1)^{\circ} \\
& V=2592.6(3) \AA^{3} \\
& Z=8 \\
& F(000)=1216 \\
& D_{\mathrm{x}}=1.553 \mathrm{Mg} \mathrm{m}^{-3}
\end{aligned}
$$

Data collection

\section{Rigaku CCD} diffractometer

Radiation source: fine-focus sealed tube Graphite monochromator $\omega$ scans

Absorption correction: multi-scan (SADABS; Sheldrick, 2004)

$T_{\min }=0.585, T_{\max }=0.809$

\section{Refinement}

Refinement on $F^{2}$

Least-squares matrix: full

$R\left[F^{2}>2 \sigma\left(F^{2}\right)\right]=0.025$

$w R\left(F^{2}\right)=0.063$

$S=1.04$

5922 reflections

331 parameters

0 restraints

Primary atom site location: structure-invariant direct methods
Mo $K \alpha$ radiation, $\lambda=0.71073 \AA$

Cell parameters from 30565 reflections

$\theta=2.2-27.5^{\circ}$

$\mu=3.16 \mathrm{~mm}^{-1}$

$T=100 \mathrm{~K}$

Rod, colourless

$0.19 \times 0.07 \times 0.07 \mathrm{~mm}$

31964 measured reflections

5922 independent reflections

5297 reflections with $I>2 \sigma(I)$

$R_{\text {int }}=0.035$

$\theta_{\max }=27.5^{\circ}, \theta_{\min }=2.2^{\circ}$

$h=-17 \rightarrow 18$

$k=-13 \rightarrow 14$

$l=-22 \rightarrow 23$

Secondary atom site location: difference Fourier map

Hydrogen site location: inferred from neighbouring sites

$\mathrm{H}$ atoms treated by a mixture of independent and constrained refinement

$w=1 /\left[\sigma^{2}\left(F_{\mathrm{o}}^{2}\right)+(0.0335 P)^{2}+1.1714 P\right]$ where $P=\left(F_{\mathrm{o}}^{2}+2 F_{\mathrm{c}}^{2}\right) / 3$

$(\Delta / \sigma)_{\max }=0.001$

$\Delta \rho_{\max }=0.56 \mathrm{e} \AA^{-3}$

$\Delta \rho_{\min }=-0.74$ e $\AA^{-3}$

\section{Special details}

Geometry. All e.s.d.'s (except the e.s.d. in the dihedral angle between two l.s. planes) are estimated using the full covariance matrix. The cell e.s.d.'s are taken into account individually in the estimation of e.s.d.'s in distances, angles and torsion angles; correlations between e.s.d.'s in cell parameters are only used when they are defined by crystal symmetry. An approximate (isotropic) treatment of cell e.s.d.'s is used for estimating e.s.d.'s involving 1.s. planes.

Refinement. Refinement of $F^{2}$ against ALL reflections. The weighted $R$-factor $w R$ and goodness of fit $S$ are based on $F^{2}$, conventional $R$-factors $R$ are based on $F$, with $F$ set to zero for negative $F^{2}$. The threshold expression of $F^{2}>\sigma\left(F^{2}\right)$ is used only for calculating $R$-factors(gt) etc. and is not relevant to the choice of reflections for refinement. $R$-factors based on $F^{2}$ are statistically about twice as large as those based on $F$, and $R$ - factors based on ALL data will be even larger.

Fractional atomic coordinates and isotropic or equivalent isotropic displacement parameters $\left(\AA^{2}\right)$

\begin{tabular}{lllll}
\hline & $x$ & $y$ & $z$ & $U_{\text {iso }} * / U_{\text {eq }}$ \\
\hline C1 & $0.18672(11)$ & $0.20670(14)$ & $0.33555(9)$ & $0.0171(3)$ \\
C2 & $0.21064(12)$ & $0.11955(16)$ & $0.39459(9)$ & $0.0208(3)$ \\
H2 & 0.2476 & 0.1419 & 0.4461 & $0.025^{*}$ \\
C3 & $0.17974(12)$ & $-0.00082(16)$ & $0.37725(10)$ & $0.0228(3)$ \\
H3 & 0.1954 & -0.0614 & 0.4171 & $0.027^{*}$ \\
C4 & $0.12604(12)$ & $-0.03303(16)$ & $0.30185(10)$ & $0.0223(3)$ \\
H4 & 0.1064 & -0.1158 & 0.2899 & $0.027^{*}$ \\
C5 & $0.10111(12)$ & $0.05592(15)$ & $0.24394(9)$ & $0.0206(3)$
\end{tabular}




\begin{tabular}{|c|c|c|c|c|}
\hline H5 & 0.0632 & 0.0334 & 0.1928 & $0.025^{*}$ \\
\hline C6 & $0.13072(11)$ & $0.17808(15)$ & $0.25938(9)$ & $0.0171(3)$ \\
\hline $\mathrm{C} 7$ & $0.10814(12)$ & $0.27030(14)$ & $0.19619(9)$ & $0.0177(3)$ \\
\hline $\mathrm{H} 7$ & 0.1615 & 0.3221 & 0.1923 & $0.021^{*}$ \\
\hline $\mathrm{C} 8$ & $0.01866(11)$ & $0.28748(14)$ & $0.14364(8)$ & $0.0156(3)$ \\
\hline C9 & $0.01101(11)$ & $0.37744(14)$ & $0.07944(9)$ & $0.0153(3)$ \\
\hline $\mathrm{C} 10$ & $-0.07591(11)$ & $0.22525(14)$ & $0.14608(9)$ & $0.0158(3)$ \\
\hline $\mathrm{C} 11$ & $-0.10312(12)$ & $0.21984(15)$ & $0.21603(9)$ & $0.0203(3)$ \\
\hline H11 & -0.0635 & 0.2605 & 0.2612 & $0.024 *$ \\
\hline $\mathrm{C} 12$ & $-0.18786(13)$ & $0.15528(16)$ & $0.21964(10)$ & $0.0234(3)$ \\
\hline H12 & -0.2057 & 0.1517 & 0.2674 & $0.028 *$ \\
\hline $\mathrm{C} 13$ & $-0.24673(12)$ & $0.09582(16)$ & $0.15388(10)$ & $0.0239(3)$ \\
\hline H13 & -0.3035 & 0.0497 & 0.1570 & $0.029 *$ \\
\hline $\mathrm{C} 14$ & $-0.22209(12)$ & $0.10418(15)$ & $0.08346(10)$ & $0.0213(3)$ \\
\hline H14 & -0.2633 & 0.0659 & 0.0379 & $0.026^{*}$ \\
\hline $\mathrm{C} 15$ & $-0.13720(12)$ & $0.16852(15)$ & $0.07977(9)$ & $0.0177(3)$ \\
\hline H15 & -0.1207 & 0.1739 & 0.0316 & $0.021 *$ \\
\hline $\mathrm{O} 1$ & $-0.06993(8)$ & $0.41209(10)$ & $0.03689(6)$ & $0.0185(2)$ \\
\hline $\mathrm{O} 2$ & $0.09775(8)$ & $0.41647(11)$ & $0.07169(7)$ & $0.0204(2)$ \\
\hline $\mathrm{H} 2 \mathrm{O}$ & $0.0845(15)$ & $0.471(2)$ & $0.0373(12)$ & $0.024 *$ \\
\hline $\mathrm{Br} 1$ & $0.230078(13)$ & $0.370757(16)$ & $0.360313(9)$ & $0.02454(6)$ \\
\hline $\mathrm{C} 16$ & $0.88556(12)$ & $0.19354(15)$ & $0.43750(9)$ & $0.0194(3)$ \\
\hline $\mathrm{C} 17$ & $0.94766(12)$ & $0.10532(16)$ & $0.41971(10)$ & $0.0234(3)$ \\
\hline H17 & 1.0158 & 0.1236 & 0.4240 & $0.028^{*}$ \\
\hline $\mathrm{C} 18$ & $0.90912(13)$ & $-0.00957(16)$ & $0.39570(10)$ & $0.0249(4)$ \\
\hline H18 & 0.9510 & -0.0708 & 0.3835 & $0.030^{*}$ \\
\hline C19 & $0.80940(13)$ & $-0.03570(15)$ & $0.38943(10)$ & $0.0227(3)$ \\
\hline H19 & 0.7834 & -0.1151 & 0.3739 & $0.027^{*}$ \\
\hline $\mathrm{C} 20$ & $0.74767(12)$ & $0.05462(15)$ & $0.40597(9)$ & $0.0204(3)$ \\
\hline $\mathrm{H} 20$ & 0.6791 & 0.0364 & 0.4003 & $0.025^{*}$ \\
\hline $\mathrm{C} 21$ & $0.78410(11)$ & $0.17179(15)$ & $0.43077(9)$ & $0.0173(3)$ \\
\hline $\mathrm{C} 22$ & $0.72086(12)$ & $0.26361(14)$ & $0.45511(9)$ & $0.0179(3)$ \\
\hline $\mathrm{H} 22$ & 0.7525 & 0.3113 & 0.4998 & $0.021^{*}$ \\
\hline $\mathrm{C} 23$ & $0.62358(12)$ & $0.28762(14)$ & $0.42138(9)$ & 0.0169 (3) \\
\hline $\mathrm{C} 24$ & $0.57101(12)$ & $0.37778(14)$ & $0.45901(9)$ & $0.0172(3)$ \\
\hline $\mathrm{C} 25$ & $0.56263(12)$ & $0.23498(14)$ & $0.34604(9)$ & $0.0173(3)$ \\
\hline $\mathrm{C} 26$ & $0.59428(12)$ & $0.24671(15)$ & $0.27832(9)$ & $0.0203(3)$ \\
\hline $\mathrm{H} 26$ & 0.6540 & 0.2906 & 0.2801 & $0.024 *$ \\
\hline $\mathrm{C} 27$ & $0.53886(13)$ & $0.19457(16)$ & $0.20854(10)$ & $0.0234(3)$ \\
\hline $\mathrm{H} 27$ & 0.5611 & 0.2024 & 0.1629 & $0.028^{*}$ \\
\hline $\mathrm{C} 28$ & $0.45100(14)$ & $0.13098(15)$ & $0.20517(10)$ & $0.0249(4)$ \\
\hline $\mathrm{H} 28$ & 0.4137 & 0.0945 & 0.1575 & $0.030^{*}$ \\
\hline $\mathrm{C} 29$ & $0.41787(13)$ & $0.12096(15)$ & $0.27187(10)$ & $0.0239(3)$ \\
\hline $\mathrm{H} 29$ & 0.3577 & 0.0779 & 0.2698 & $0.029^{*}$ \\
\hline $\mathrm{C} 30$ & $0.47260(12)$ & $0.17383(15)$ & $0.34140(10)$ & $0.0201(3)$ \\
\hline H30 & 0.4487 & 0.1685 & 0.3864 & $0.024^{*}$ \\
\hline $\mathrm{O} 3$ & $0.48613(8)$ & $0.41328(11)$ & $0.42624(6)$ & $0.0207(2)$ \\
\hline $\mathrm{O} 4$ & $0.62187(9)$ & $0.41561(11)$ & $0.52942(6)$ & $0.0207(2)$ \\
\hline
\end{tabular}




$\begin{array}{lllll}\mathrm{H} 4 \mathrm{O} & 0.5877(16) & 0.467(2) & 0.5428(12) & 0.025^{*} \\ \mathrm{Br} 2 & 0.942911(13) & 0.350346(17) & 0.471076(12) & 0.03017(6)\end{array}$

Atomic displacement parameters $\left(\AA^{2}\right)$

\begin{tabular}{|c|c|c|c|c|c|c|}
\hline & $U^{11}$ & $U^{22}$ & $U^{33}$ & $U^{12}$ & $U^{13}$ & $U^{23}$ \\
\hline $\mathrm{C} 1$ & $0.0130(7)$ & $0.0176(7)$ & $0.0209(7)$ & $-0.0002(6)$ & $0.0049(6)$ & $0.0010(6)$ \\
\hline $\mathrm{C} 2$ & $0.0144(7)$ & $0.0290(9)$ & $0.0184(7)$ & $0.0024(6)$ & $0.0034(6)$ & $0.0036(6)$ \\
\hline $\mathrm{C} 3$ & $0.0168(7)$ & $0.0269(9)$ & $0.0254(8)$ & $0.0037(6)$ & $0.0071(6)$ & $0.0107(7)$ \\
\hline $\mathrm{C} 4$ & $0.0187(8)$ & $0.0179(8)$ & $0.0308(9)$ & $0.0006(6)$ & $0.0075(7)$ & $0.0030(6)$ \\
\hline $\mathrm{C} 5$ & $0.0175(7)$ & $0.0228(8)$ & $0.0207(8)$ & $0.0006(6)$ & $0.0039(6)$ & $0.0007(6)$ \\
\hline C6 & $0.0131(7)$ & $0.0200(8)$ & $0.0181(7)$ & $0.0014(6)$ & $0.0041(6)$ & $0.0013(6)$ \\
\hline $\mathrm{C} 7$ & $0.0180(7)$ & $0.0179(8)$ & $0.0176(7)$ & $-0.0003(6)$ & $0.0055(6)$ & $-0.0008(6)$ \\
\hline C8 & $0.0171(7)$ & $0.0156(7)$ & $0.0152(7)$ & $0.0002(6)$ & $0.0061(6)$ & $0.0000(5)$ \\
\hline C9 & $0.0156(7)$ & $0.0164(7)$ & $0.0147(7)$ & $-0.0006(6)$ & $0.0056(6)$ & $-0.0020(5)$ \\
\hline $\mathrm{C} 10$ & $0.0147(7)$ & $0.0160(7)$ & $0.0174(7)$ & $0.0027(6)$ & $0.0057(6)$ & $0.0029(6)$ \\
\hline C11 & $0.0192(8)$ & $0.0246(8)$ & $0.0171(7)$ & $0.0031(6)$ & $0.0053(6)$ & $0.0023(6)$ \\
\hline $\mathrm{C} 12$ & $0.0214(8)$ & $0.0298(9)$ & $0.0224(8)$ & $0.0042(7)$ & $0.0117(7)$ & $0.0068(7)$ \\
\hline $\mathrm{C} 13$ & $0.0184(8)$ & $0.0239(9)$ & $0.0320(9)$ & $-0.0010(6)$ & $0.0112(7)$ & $0.0063(7)$ \\
\hline $\mathrm{C} 14$ & $0.0188(8)$ & $0.0202(8)$ & $0.0247(8)$ & $-0.0009(6)$ & $0.0057(6)$ & $-0.0010(6)$ \\
\hline $\mathrm{C} 15$ & $0.0184(7)$ & $0.0180(7)$ & $0.0179(7)$ & $0.0013(6)$ & $0.0070(6)$ & $0.0008(6)$ \\
\hline $\mathrm{O} 1$ & $0.0147(5)$ & $0.0218(6)$ & $0.0185(5)$ & $0.0001(4)$ & $0.0036(4)$ & $0.0037(4)$ \\
\hline $\mathrm{O} 2$ & $0.0143(5)$ & $0.0249(6)$ & $0.0224(6)$ & $0.0001(4)$ & 0.0058 (4) & $0.0086(5)$ \\
\hline $\mathrm{Br} 1$ & $0.02498(9)$ & $0.02182(9)$ & $0.02174(9)$ & $-0.00288(6)$ & $-0.00199(6)$ & $-0.00055(6)$ \\
\hline $\mathrm{C} 16$ & $0.0192(7)$ & $0.0187(8)$ & $0.0193(7)$ & $-0.0005(6)$ & $0.0040(6)$ & $0.0007(6)$ \\
\hline $\mathrm{C} 17$ & $0.0173(8)$ & $0.0286(9)$ & $0.0248(8)$ & $0.0035(7)$ & $0.0069(6)$ & $0.0019(7)$ \\
\hline $\mathrm{C} 18$ & $0.0243(9)$ & $0.0246(9)$ & $0.0276(9)$ & $0.0090(7)$ & $0.0100(7)$ & $0.0008(7)$ \\
\hline C19 & $0.0270(8)$ & $0.0168(8)$ & $0.0261(8)$ & $0.0010(6)$ & $0.0103(7)$ & $-0.0001(6)$ \\
\hline $\mathrm{C} 20$ & $0.0197(8)$ & $0.0213(8)$ & $0.0219(8)$ & $-0.0003(6)$ & $0.0085(6)$ & $0.0026(6)$ \\
\hline $\mathrm{C} 21$ & $0.0174(7)$ & $0.0204(8)$ & $0.0146(7)$ & $0.0019(6)$ & $0.0051(6)$ & $0.0028(6)$ \\
\hline $\mathrm{C} 22$ & $0.0188(7)$ & $0.0182(8)$ & $0.0177(7)$ & $-0.0008(6)$ & $0.0070(6)$ & $0.0002(6)$ \\
\hline $\mathrm{C} 23$ & $0.0187(7)$ & $0.0158(7)$ & $0.0186(7)$ & $-0.0005(6)$ & $0.0091(6)$ & $0.0011(6)$ \\
\hline $\mathrm{C} 24$ & $0.0181(7)$ & $0.0171(7)$ & $0.0180(7)$ & $-0.0016(6)$ & $0.0079(6)$ & $0.0015(6)$ \\
\hline $\mathrm{C} 25$ & $0.0184(7)$ & $0.0150(7)$ & $0.0192(7)$ & $0.0030(6)$ & $0.0063(6)$ & $-0.0001(6)$ \\
\hline $\mathrm{C} 26$ & $0.0200(8)$ & $0.0202(8)$ & $0.0222(8)$ & $0.0016(6)$ & $0.0085(6)$ & $0.0007(6)$ \\
\hline $\mathrm{C} 27$ & $0.0274(9)$ & $0.0240(9)$ & $0.0205(8)$ & $0.0034(7)$ & $0.0097(7)$ & $-0.0004(6)$ \\
\hline $\mathrm{C} 28$ & $0.0294(9)$ & $0.0211(8)$ & $0.0224(8)$ & $0.0012(7)$ & $0.0044(7)$ & $-0.0052(6)$ \\
\hline C29 & $0.0227(8)$ & $0.0196(8)$ & $0.0297(9)$ & $-0.0033(6)$ & $0.0077(7)$ & $-0.0029(7)$ \\
\hline $\mathrm{C} 30$ & $0.0219(8)$ & $0.0180(8)$ & $0.0228(8)$ & $0.0005(6)$ & $0.0099(6)$ & $-0.0003(6)$ \\
\hline $\mathrm{O} 3$ & $0.0174(5)$ & $0.0240(6)$ & $0.0207(5)$ & $0.0026(5)$ & $0.0052(4)$ & $-0.0035(5)$ \\
\hline $\mathrm{O} 4$ & $0.0193(6)$ & $0.0233(6)$ & $0.0195(5)$ & $0.0049(5)$ & $0.0054(4)$ & $-0.0045(5)$ \\
\hline $\mathrm{Br} 2$ & $0.02001(9)$ & $0.02494(10)$ & $0.04386(12)$ & $-0.00432(6)$ & $0.00599(8)$ & $-0.00679(7)$ \\
\hline
\end{tabular}

Geometric parameters $\left(A,{ }^{o}\right)$

\begin{tabular}{llll}
\hline $\mathrm{C} 1-\mathrm{C} 2$ & $1.388(2)$ & $\mathrm{C} 16-\mathrm{C} 17$ & $1.386(2)$ \\
$\mathrm{C} 1-\mathrm{C} 6$ & $1.400(2)$ & $\mathrm{C} 16-\mathrm{C} 21$ & $1.401(2)$ \\
$\mathrm{C} 1-\mathrm{Br} 1$ & $1.9002(16)$ & $\mathrm{C} 16-\mathrm{Br} 2$ & $1.9111(16)$
\end{tabular}




\begin{tabular}{|c|c|c|c|}
\hline $\mathrm{C} 2-\mathrm{C} 3$ & $1.389(2)$ & $\mathrm{C} 17-\mathrm{C} 18$ & $1.383(3)$ \\
\hline $\mathrm{C} 2-\mathrm{H} 2$ & 0.9500 & C17-H17 & 0.9500 \\
\hline $\mathrm{C} 3-\mathrm{C} 4$ & $1.389(2)$ & $\mathrm{C} 18-\mathrm{C} 19$ & $1.388(2)$ \\
\hline $\mathrm{C} 3-\mathrm{H} 3$ & 0.9500 & $\mathrm{C} 18-\mathrm{H} 18$ & 0.9500 \\
\hline $\mathrm{C} 4-\mathrm{C} 5$ & $1.388(2)$ & $\mathrm{C} 19-\mathrm{C} 20$ & $1.390(2)$ \\
\hline $\mathrm{C} 4-\mathrm{H} 4$ & 0.9500 & C19-H19 & 0.9500 \\
\hline $\mathrm{C} 5-\mathrm{C} 6$ & $1.399(2)$ & $\mathrm{C} 20-\mathrm{C} 21$ & $1.400(2)$ \\
\hline $\mathrm{C} 5-\mathrm{H} 5$ & 0.9500 & $\mathrm{C} 20-\mathrm{H} 20$ & 0.9500 \\
\hline $\mathrm{C} 6-\mathrm{C} 7$ & $1.477(2)$ & $\mathrm{C} 21-\mathrm{C} 22$ & $1.474(2)$ \\
\hline $\mathrm{C} 7-\mathrm{C} 8$ & $1.346(2)$ & $\mathrm{C} 22-\mathrm{C} 23$ & $1.344(2)$ \\
\hline $\mathrm{C} 7-\mathrm{H} 7$ & 0.9500 & $\mathrm{C} 22-\mathrm{H} 22$ & 0.9500 \\
\hline $\mathrm{C} 8-\mathrm{C} 9$ & $1.488(2)$ & $\mathrm{C} 23-\mathrm{C} 25$ & $1.489(2)$ \\
\hline $\mathrm{C} 8-\mathrm{C} 10$ & $1.490(2)$ & $\mathrm{C} 23-\mathrm{C} 24$ & $1.490(2)$ \\
\hline $\mathrm{C} 9-\mathrm{O} 1$ & $1.2287(18)$ & $\mathrm{C} 24-\mathrm{O} 3$ & 1.2247 (19) \\
\hline $\mathrm{C} 9-\mathrm{O} 2$ & $1.3205(18)$ & $\mathrm{C} 24-\mathrm{O} 4$ & $1.3236(19)$ \\
\hline $\mathrm{C} 10-\mathrm{C} 15$ & $1.395(2)$ & $\mathrm{C} 25-\mathrm{C} 30$ & $1.399(2)$ \\
\hline $\mathrm{C} 10-\mathrm{C} 11$ & $1.400(2)$ & $\mathrm{C} 25-\mathrm{C} 26$ & $1.399(2)$ \\
\hline $\mathrm{C} 11-\mathrm{C} 12$ & $1.388(2)$ & $\mathrm{C} 26-\mathrm{C} 27$ & $1.390(2)$ \\
\hline $\mathrm{C} 11-\mathrm{H} 11$ & 0.9500 & $\mathrm{C} 26-\mathrm{H} 26$ & 0.9500 \\
\hline $\mathrm{C} 12-\mathrm{C} 13$ & $1.390(3)$ & $\mathrm{C} 27-\mathrm{C} 28$ & $1.390(2)$ \\
\hline $\mathrm{C} 12-\mathrm{H} 12$ & 0.9500 & $\mathrm{C} 27-\mathrm{H} 27$ & 0.9500 \\
\hline $\mathrm{C} 13-\mathrm{C} 14$ & $1.391(2)$ & $\mathrm{C} 28-\mathrm{C} 29$ & $1.392(2)$ \\
\hline $\mathrm{C} 13-\mathrm{H} 13$ & 0.9500 & $\mathrm{C} 28-\mathrm{H} 28$ & 0.9500 \\
\hline $\mathrm{C} 14-\mathrm{C} 15$ & $1.389(2)$ & $\mathrm{C} 29-\mathrm{C} 30$ & $1.387(2)$ \\
\hline $\mathrm{C} 14-\mathrm{H} 14$ & 0.9500 & $\mathrm{C} 29-\mathrm{H} 29$ & 0.9500 \\
\hline $\mathrm{C} 15-\mathrm{H} 15$ & 0.9500 & $\mathrm{C} 30-\mathrm{H} 30$ & 0.9500 \\
\hline $\mathrm{O} 2-\mathrm{H} 2 \mathrm{O}$ & $0.84(2)$ & $\mathrm{O} 4-\mathrm{H} 4 \mathrm{O}$ & $0.81(2)$ \\
\hline $\mathrm{C} 2-\mathrm{C} 1-\mathrm{C} 6$ & $122.28(15)$ & $\mathrm{C} 17-\mathrm{C} 16-\mathrm{C} 21$ & $122.53(15)$ \\
\hline $\mathrm{C} 2-\mathrm{C} 1-\mathrm{Br} 1$ & $118.35(12)$ & $\mathrm{C} 17-\mathrm{C} 16-\mathrm{Br} 2$ & $117.43(12)$ \\
\hline $\mathrm{C} 6-\mathrm{C} 1-\mathrm{Br} 1$ & $119.36(12)$ & $\mathrm{C} 21-\mathrm{C} 16-\mathrm{Br} 2$ & $120.03(12)$ \\
\hline $\mathrm{C} 1-\mathrm{C} 2-\mathrm{C} 3$ & $118.96(15)$ & $\mathrm{C} 18-\mathrm{C} 17-\mathrm{C} 16$ & $119.17(15)$ \\
\hline $\mathrm{C} 1-\mathrm{C} 2-\mathrm{H} 2$ & 120.5 & $\mathrm{C} 18-\mathrm{C} 17-\mathrm{H} 17$ & 120.4 \\
\hline $\mathrm{C} 3-\mathrm{C} 2-\mathrm{H} 2$ & 120.5 & $\mathrm{C} 16-\mathrm{C} 17-\mathrm{H} 17$ & 120.4 \\
\hline $\mathrm{C} 4-\mathrm{C} 3-\mathrm{C} 2$ & $120.28(15)$ & $\mathrm{C} 17-\mathrm{C} 18-\mathrm{C} 19$ & $120.21(15)$ \\
\hline $\mathrm{C} 4-\mathrm{C} 3-\mathrm{H} 3$ & 119.9 & $\mathrm{C} 17-\mathrm{C} 18-\mathrm{H} 18$ & 119.9 \\
\hline $\mathrm{C} 2-\mathrm{C} 3-\mathrm{H} 3$ & 119.9 & $\mathrm{C} 19-\mathrm{C} 18-\mathrm{H} 18$ & 119.9 \\
\hline $\mathrm{C} 5-\mathrm{C} 4-\mathrm{C} 3$ & $119.91(16)$ & $\mathrm{C} 18-\mathrm{C} 19-\mathrm{C} 20$ & $119.86(16)$ \\
\hline $\mathrm{C} 5-\mathrm{C} 4-\mathrm{H} 4$ & 120.0 & $\mathrm{C} 18-\mathrm{C} 19-\mathrm{H} 19$ & 120.1 \\
\hline $\mathrm{C} 3-\mathrm{C} 4-\mathrm{H} 4$ & 120.0 & $\mathrm{C} 20-\mathrm{C} 19-\mathrm{H} 19$ & 120.1 \\
\hline $\mathrm{C} 4-\mathrm{C} 5-\mathrm{C} 6$ & $121.33(15)$ & $\mathrm{C} 19-\mathrm{C} 20-\mathrm{C} 21$ & $121.55(15)$ \\
\hline $\mathrm{C} 4-\mathrm{C} 5-\mathrm{H} 5$ & 119.3 & $\mathrm{C} 19-\mathrm{C} 20-\mathrm{H} 20$ & 119.2 \\
\hline $\mathrm{C} 6-\mathrm{C} 5-\mathrm{H} 5$ & 119.3 & $\mathrm{C} 21-\mathrm{C} 20-\mathrm{H} 20$ & 119.2 \\
\hline $\mathrm{C} 5-\mathrm{C} 6-\mathrm{C} 1$ & $117.21(14)$ & $\mathrm{C} 20-\mathrm{C} 21-\mathrm{C} 16$ & $116.66(15)$ \\
\hline $\mathrm{C} 5-\mathrm{C} 6-\mathrm{C} 7$ & $120.64(14)$ & $\mathrm{C} 20-\mathrm{C} 21-\mathrm{C} 22$ & $121.33(14)$ \\
\hline $\mathrm{C} 1-\mathrm{C} 6-\mathrm{C} 7$ & $122.06(14)$ & $\mathrm{C} 16-\mathrm{C} 21-\mathrm{C} 22$ & $121.80(15)$ \\
\hline $\mathrm{C} 8-\mathrm{C} 7-\mathrm{C} 6$ & $125.82(14)$ & $\mathrm{C} 23-\mathrm{C} 22-\mathrm{C} 21$ & $127.45(15)$ \\
\hline $\mathrm{C} 8-\mathrm{C} 7-\mathrm{H} 7$ & 117.1 & $\mathrm{C} 23-\mathrm{C} 22-\mathrm{H} 22$ & 116.3 \\
\hline
\end{tabular}




\begin{tabular}{|c|c|c|c|}
\hline $\mathrm{C} 6-\mathrm{C} 7-\mathrm{H} 7$ & 117.1 & $\mathrm{C} 21-\mathrm{C} 22-\mathrm{H} 22$ & 116.3 \\
\hline $\mathrm{C} 7-\mathrm{C} 8-\mathrm{C} 9$ & $118.81(14)$ & $\mathrm{C} 22-\mathrm{C} 23-\mathrm{C} 25$ & $125.33(14)$ \\
\hline $\mathrm{C} 7-\mathrm{C} 8-\mathrm{C} 10$ & $124.62(14)$ & $\mathrm{C} 22-\mathrm{C} 23-\mathrm{C} 24$ & $118.99(14)$ \\
\hline $\mathrm{C} 9-\mathrm{C} 8-\mathrm{C} 10$ & $116.54(13)$ & $\mathrm{C} 25-\mathrm{C} 23-\mathrm{C} 24$ & $115.63(13)$ \\
\hline $\mathrm{O} 1-\mathrm{C} 9-\mathrm{O} 2$ & $122.81(14)$ & $\mathrm{O} 3-\mathrm{C} 24-\mathrm{O} 4$ & $122.96(14)$ \\
\hline $\mathrm{O} 1-\mathrm{C} 9-\mathrm{C} 8$ & $122.37(13)$ & $\mathrm{O} 3-\mathrm{C} 24-\mathrm{C} 23$ & $121.41(14)$ \\
\hline $\mathrm{O} 2-\mathrm{C} 9-\mathrm{C} 8$ & $114.82(13)$ & $\mathrm{O} 4-\mathrm{C} 24-\mathrm{C} 23$ & $115.63(13)$ \\
\hline $\mathrm{C} 15-\mathrm{C} 10-\mathrm{C} 11$ & $118.89(14)$ & $\mathrm{C} 30-\mathrm{C} 25-\mathrm{C} 26$ & $118.78(14)$ \\
\hline $\mathrm{C} 15-\mathrm{C} 10-\mathrm{C} 8$ & $120.92(13)$ & $\mathrm{C} 30-\mathrm{C} 25-\mathrm{C} 23$ & $120.85(13)$ \\
\hline $\mathrm{C} 11-\mathrm{C} 10-\mathrm{C} 8$ & $120.17(14)$ & $\mathrm{C} 26-\mathrm{C} 25-\mathrm{C} 23$ & $120.37(14)$ \\
\hline $\mathrm{C} 12-\mathrm{C} 11-\mathrm{C} 10$ & $120.26(15)$ & $\mathrm{C} 27-\mathrm{C} 26-\mathrm{C} 25$ & $120.34(15)$ \\
\hline $\mathrm{C} 12-\mathrm{C} 11-\mathrm{H} 11$ & 119.9 & $\mathrm{C} 27-\mathrm{C} 26-\mathrm{H} 26$ & 119.8 \\
\hline $\mathrm{C} 10-\mathrm{C} 11-\mathrm{H} 11$ & 119.9 & $\mathrm{C} 25-\mathrm{C} 26-\mathrm{H} 26$ & 119.8 \\
\hline $\mathrm{C} 11-\mathrm{C} 12-\mathrm{C} 13$ & $120.41(15)$ & $\mathrm{C} 26-\mathrm{C} 27-\mathrm{C} 28$ & $120.33(15)$ \\
\hline $\mathrm{C} 11-\mathrm{C} 12-\mathrm{H} 12$ & 119.8 & $\mathrm{C} 26-\mathrm{C} 27-\mathrm{H} 27$ & 119.8 \\
\hline $\mathrm{C} 13-\mathrm{C} 12-\mathrm{H} 12$ & 119.8 & $\mathrm{C} 28-\mathrm{C} 27-\mathrm{H} 27$ & 119.8 \\
\hline $\mathrm{C} 12-\mathrm{C} 13-\mathrm{C} 14$ & $119.68(15)$ & $\mathrm{C} 27-\mathrm{C} 28-\mathrm{C} 29$ & $119.73(16)$ \\
\hline $\mathrm{C} 12-\mathrm{C} 13-\mathrm{H} 13$ & 120.2 & $\mathrm{C} 27-\mathrm{C} 28-\mathrm{H} 28$ & 120.1 \\
\hline $\mathrm{C} 14-\mathrm{C} 13-\mathrm{H} 13$ & 120.2 & $\mathrm{C} 29-\mathrm{C} 28-\mathrm{H} 28$ & 120.1 \\
\hline $\mathrm{C} 15-\mathrm{C} 14-\mathrm{C} 13$ & $119.96(16)$ & $\mathrm{C} 30-\mathrm{C} 29-\mathrm{C} 28$ & $120.04(16)$ \\
\hline $\mathrm{C} 15-\mathrm{C} 14-\mathrm{H} 14$ & 120.0 & $\mathrm{C} 30-\mathrm{C} 29-\mathrm{H} 29$ & 120.0 \\
\hline $\mathrm{C} 13-\mathrm{C} 14-\mathrm{H} 14$ & 120.0 & $\mathrm{C} 28-\mathrm{C} 29-\mathrm{H} 29$ & 120.0 \\
\hline $\mathrm{C} 14-\mathrm{C} 15-\mathrm{C} 10$ & $120.74(14)$ & $\mathrm{C} 29-\mathrm{C} 30-\mathrm{C} 25$ & $120.73(15)$ \\
\hline $\mathrm{C} 14-\mathrm{C} 15-\mathrm{H} 15$ & 119.6 & $\mathrm{C} 29-\mathrm{C} 30-\mathrm{H} 30$ & 119.6 \\
\hline $\mathrm{C} 10-\mathrm{C} 15-\mathrm{H} 15$ & 119.6 & $\mathrm{C} 25-\mathrm{C} 30-\mathrm{H} 30$ & 119.6 \\
\hline $\mathrm{C} 9-\mathrm{O} 2-\mathrm{H} 2 \mathrm{O}$ & $106.5(14)$ & $\mathrm{C} 24-\mathrm{O} 4-\mathrm{H} 4 \mathrm{O}$ & $106.9(14)$ \\
\hline $\mathrm{C} 6-\mathrm{C} 1-\mathrm{C} 2-\mathrm{C} 3$ & $1.3(2)$ & $\mathrm{C} 21-\mathrm{C} 16-\mathrm{C} 17-\mathrm{C} 18$ & $-1.2(3)$ \\
\hline $\mathrm{Br} 1-\mathrm{C} 1-\mathrm{C} 2-\mathrm{C} 3$ & $-179.76(12)$ & $\mathrm{Br} 2-\mathrm{C} 16-\mathrm{C} 17-\mathrm{C} 18$ & $179.57(13)$ \\
\hline $\mathrm{C} 1-\mathrm{C} 2-\mathrm{C} 3-\mathrm{C} 4$ & $0.2(2)$ & $\mathrm{C} 16-\mathrm{C} 17-\mathrm{C} 18-\mathrm{C} 19$ & $0.1(3)$ \\
\hline $\mathrm{C} 2-\mathrm{C} 3-\mathrm{C} 4-\mathrm{C} 5$ & $-1.5(2)$ & $\mathrm{C} 17-\mathrm{C} 18-\mathrm{C} 19-\mathrm{C} 20$ & $1.2(3)$ \\
\hline $\mathrm{C} 3-\mathrm{C} 4-\mathrm{C} 5-\mathrm{C} 6$ & $1.3(2)$ & $\mathrm{C} 18-\mathrm{C} 19-\mathrm{C} 20-\mathrm{C} 21$ & $-1.5(2)$ \\
\hline $\mathrm{C} 4-\mathrm{C} 5-\mathrm{C} 6-\mathrm{C} 1$ & $0.2(2)$ & $\mathrm{C} 19-\mathrm{C} 20-\mathrm{C} 21-\mathrm{C} 16$ & $0.4(2)$ \\
\hline $\mathrm{C} 4-\mathrm{C} 5-\mathrm{C} 6-\mathrm{C} 7$ & $176.79(14)$ & $\mathrm{C} 19-\mathrm{C} 20-\mathrm{C} 21-\mathrm{C} 22$ & $-174.34(15)$ \\
\hline $\mathrm{C} 2-\mathrm{C} 1-\mathrm{C} 6-\mathrm{C} 5$ & $-1.5(2)$ & $\mathrm{C} 17-\mathrm{C} 16-\mathrm{C} 21-\mathrm{C} 20$ & $0.9(2)$ \\
\hline $\mathrm{Br} 1-\mathrm{C} 1-\mathrm{C} 6-\mathrm{C} 5$ & $179.60(11)$ & $\mathrm{Br} 2-\mathrm{C} 16-\mathrm{C} 21-\mathrm{C} 20$ & $-179.89(11)$ \\
\hline $\mathrm{C} 2-\mathrm{C} 1-\mathrm{C} 6-\mathrm{C} 7$ & $-178.07(14)$ & $\mathrm{C} 17-\mathrm{C} 16-\mathrm{C} 21-\mathrm{C} 22$ & $175.67(15)$ \\
\hline $\mathrm{Br} 1-\mathrm{C} 1-\mathrm{C} 6-\mathrm{C} 7$ & $3.0(2)$ & $\mathrm{Br} 2-\mathrm{C} 16-\mathrm{C} 21-\mathrm{C} 22$ & $-5.1(2)$ \\
\hline $\mathrm{C} 5-\mathrm{C} 6-\mathrm{C} 7-\mathrm{C} 8$ & $48.0(2)$ & $\mathrm{C} 20-\mathrm{C} 21-\mathrm{C} 22-\mathrm{C} 23$ & $-41.2(2)$ \\
\hline $\mathrm{C} 1-\mathrm{C} 6-\mathrm{C} 7-\mathrm{C} 8$ & $-135.58(17)$ & $\mathrm{C} 16-\mathrm{C} 21-\mathrm{C} 22-\mathrm{C} 23$ & $144.30(17)$ \\
\hline $\mathrm{C} 6-\mathrm{C} 7-\mathrm{C} 8-\mathrm{C} 9$ & $-174.75(14)$ & $\mathrm{C} 21-\mathrm{C} 22-\mathrm{C} 23-\mathrm{C} 25$ & $-7.6(3)$ \\
\hline $\mathrm{C} 6-\mathrm{C} 7-\mathrm{C} 8-\mathrm{C} 10$ & $7.3(2)$ & $\mathrm{C} 21-\mathrm{C} 22-\mathrm{C} 23-\mathrm{C} 24$ & $175.15(14)$ \\
\hline $\mathrm{C} 7-\mathrm{C} 8-\mathrm{C} 9-\mathrm{O} 1$ & $-168.38(15)$ & $\mathrm{C} 22-\mathrm{C} 23-\mathrm{C} 24-\mathrm{O} 3$ & $171.51(15)$ \\
\hline $\mathrm{C} 10-\mathrm{C} 8-\mathrm{C} 9-\mathrm{O} 1$ & $9.7(2)$ & $\mathrm{C} 25-\mathrm{C} 23-\mathrm{C} 24-\mathrm{O} 3$ & $-6.0(2)$ \\
\hline $\mathrm{C} 7-\mathrm{C} 8-\mathrm{C} 9-\mathrm{O} 2$ & $11.9(2)$ & $\mathrm{C} 22-\mathrm{C} 23-\mathrm{C} 24-\mathrm{O} 4$ & $-8.7(2)$ \\
\hline $\mathrm{C} 10-\mathrm{C} 8-\mathrm{C} 9-\mathrm{O} 2$ & $-169.98(13)$ & $\mathrm{C} 25-\mathrm{C} 23-\mathrm{C} 24-\mathrm{O} 4$ & $173.74(13)$ \\
\hline $\mathrm{C} 7-\mathrm{C} 8-\mathrm{C} 10-\mathrm{C} 15$ & $-131.17(17)$ & $\mathrm{C} 22-\mathrm{C} 23-\mathrm{C} 25-\mathrm{C} 30$ & $125.69(17)$ \\
\hline $\mathrm{C} 9-\mathrm{C} 8-\mathrm{C} 10-\mathrm{C} 15$ & $50.8(2)$ & $\mathrm{C} 24-\mathrm{C} 23-\mathrm{C} 25-\mathrm{C} 30$ & $-57.0(2)$ \\
\hline
\end{tabular}




$\begin{array}{ll}\mathrm{C} 7-\mathrm{C} 8-\mathrm{C} 10-\mathrm{C} 11 & 47.0(2) \\ \mathrm{C} 9-\mathrm{C} 8-\mathrm{C} 10-\mathrm{C} 11 & -131.04(15) \\ \mathrm{C} 15-\mathrm{C} 10-\mathrm{C} 11-\mathrm{C} 12 & 2.3(2) \\ \mathrm{C} 8-\mathrm{C} 10-\mathrm{C} 11-\mathrm{C} 12 & -175.92(15) \\ \mathrm{C} 10-\mathrm{C} 11-\mathrm{C} 12-\mathrm{C} 13 & -0.3(3) \\ \mathrm{C} 11-\mathrm{C} 12-\mathrm{C} 13-\mathrm{C} 14 & -1.9(3) \\ \mathrm{C} 12-\mathrm{C} 13-\mathrm{C} 14-\mathrm{C} 15 & 2.0(3) \\ \mathrm{C} 13-\mathrm{C} 14-\mathrm{C} 15-\mathrm{C} 10 & 0.0(2) \\ \mathrm{C} 11-\mathrm{C} 10-\mathrm{C} 15-\mathrm{C} 14 & -2.1(2) \\ \mathrm{C} 8-\mathrm{C} 10-\mathrm{C} 15-\mathrm{C} 14 & 176.04(14)\end{array}$

$\mathrm{C} 22-\mathrm{C} 23-\mathrm{C} 25-\mathrm{C} 26$
$\mathrm{C} 24-\mathrm{C} 23-\mathrm{C} 25-\mathrm{C} 26$
$\mathrm{C} 30-\mathrm{C} 25-\mathrm{C} 26-\mathrm{C} 27$
$\mathrm{C} 23-\mathrm{C} 25-\mathrm{C} 26-\mathrm{C} 27$
$\mathrm{C} 25-\mathrm{C} 26-\mathrm{C} 27-\mathrm{C} 28$
$\mathrm{C} 26-\mathrm{C} 27-\mathrm{C} 28-\mathrm{C} 29$
$\mathrm{C} 27-\mathrm{C} 28-\mathrm{C} 29-\mathrm{C} 30$
$\mathrm{C} 28-\mathrm{C} 29-\mathrm{C} 30-\mathrm{C} 25$
$\mathrm{C} 26-\mathrm{C} 25-\mathrm{C} 30-\mathrm{C} 29$
$\mathrm{C} 23-\mathrm{C} 25-\mathrm{C} 30-\mathrm{C} 29$

$-54.8(2)$

$122.57(16)$

$-2.3(2)$

$178.17(15)$

$0.5(2)$

$0.8(3)$

$-0.3(3)$

$-1.6(3)$

$2.9(2)$

$-177.60(15)$

Hydrogen-bond geometry $\left(\AA,{ }^{\circ}\right)$

\begin{tabular}{lllll}
\hline$D-\mathrm{H} \cdots A$ & $D-\mathrm{H}$ & $\mathrm{H} \cdots A$ & $D \cdots A$ & $D-\mathrm{H} \cdots A$ \\
\hline $\mathrm{O} 2-\mathrm{H} 2 O \cdots \mathrm{O} 1^{\mathrm{i}}$ & $0.84(2)$ & $1.80(2)$ & $2.6402(16)$ & $174(2)$ \\
$\mathrm{O} 4-\mathrm{H} 4 O \cdots 3^{3 i}$ & $0.81(2)$ & $1.84(2)$ & $2.6478(16)$ & $178(2)$ \\
$\mathrm{C} 5-\mathrm{H} 5 \cdots \mathrm{O} 33^{i i i}$ & 0.95 & 2.42 & $3.323(2)$ & 158 \\
$\mathrm{C} 20-\mathrm{H} 20 \cdots \mathrm{O} 1^{\mathrm{iii}}$ & 0.95 & 2.52 & $3.3072(19)$ & 141
\end{tabular}

Symmetry codes: (i) $-x,-y+1,-z$; (ii) $-x+1,-y+1,-z+1$; (iii) $-x+1 / 2, y-1 / 2,-z+1 / 2$. 